\title{
\#USGS
}

science for a changing world

Prepared in cooperation with the Mason Conservation District

\section{Groundwater and Surface-Water Data Collection for Mason County, Western Washington, 2016-18}

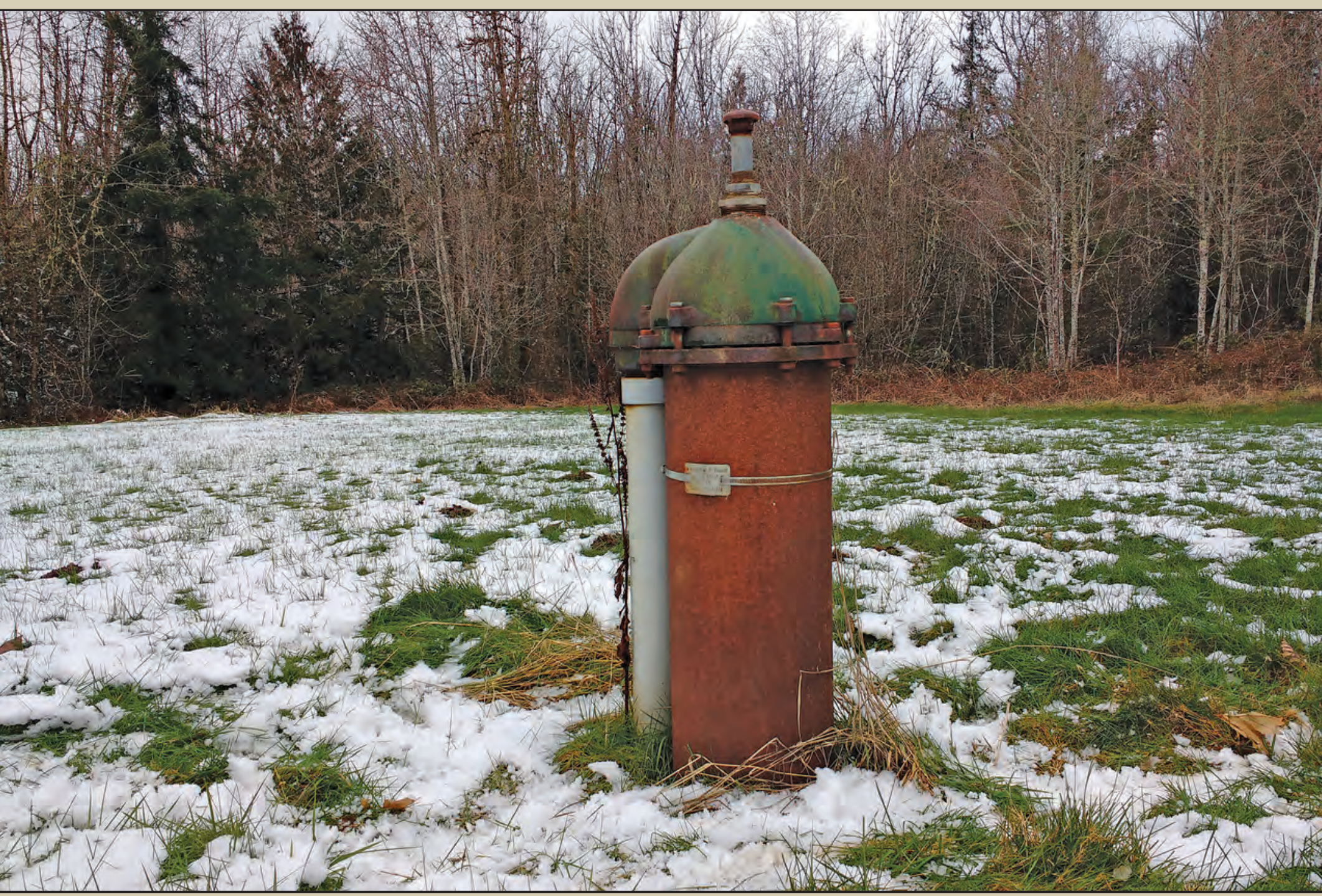

Data Series 1106

U.S. Department of the Interior

U.S. Geological Survey 
Cover: Wellhead at U.S. Geological Survey site 20N/03W-31J01 near Shelton, Mason County, Washington, February 23, 2018. 


\section{Groundwater and Surface-Water Data Collection for Mason County, Western Washington, 2016-18}

By Alison E. Tecca and Lonna M. Frans

Prepared in cooperation with the Mason Conservation District

Data Series 1106 


\title{
U.S. Department of the Interior DAVID BERNHARDT, Acting Secretary
}

\section{U.S. Geological Survey James F. Reilly II, Director}

\author{
U.S. Geological Survey, Reston, Virginia: 2019
}

For more information on the USGS - the Federal source for science about the Earth, its natural and living resources, natural hazards, and the environment-visit https://www.usgs.gov or call 1-888-ASK-USGS.

For an overview of USGS information products, including maps, imagery, and publications, visit https://store.usgs.gov.

Any use of trade, firm, or product names is for descriptive purposes only and does not imply endorsement by the U.S. Government.

Although this information product, for the most part, is in the public domain, it also may contain copyrighted materials as noted in the text. Permission to reproduce copyrighted items must be secured from the copyright owner.

Suggested citation:

Tecca, A.E., and Frans, L.M., 2019, Groundwater and surface-water data collection for Mason County, western Washington, 2016-18: U.S. Geological Survey Data Series 1106, 26 p., https://doi.org/10.3133/ds1106.

ISSN 2327-638X (online) 


\section{Contents}

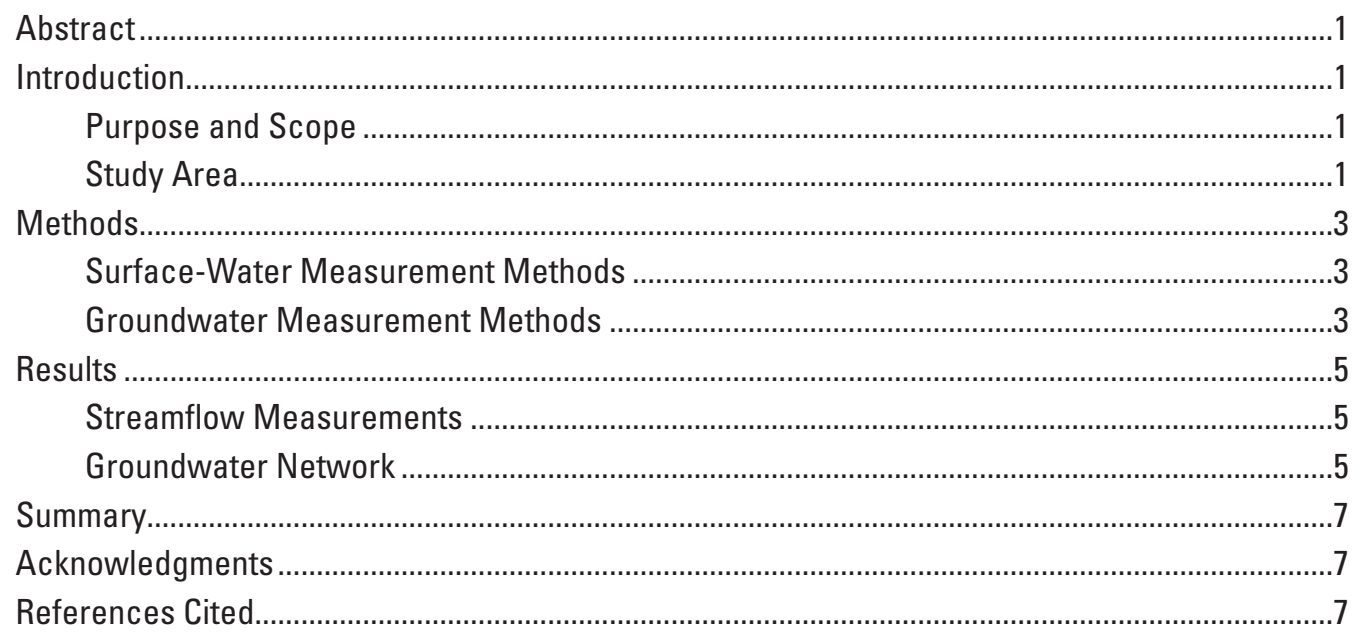

\section{Figures}

1. Map showing the general project study area, Mason County, western Washington, 2016-18.

2. Map showing surface-water site locations, Mason County, western Washington, 2016-18

3. Map showing groundwater site locations, Mason County, western Washington,

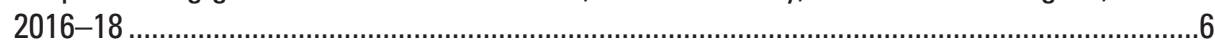

4. Examples of hydrographs for monthly water-level wells, Mason County, western Washington, 2016-18.

\section{Tables}

1. Miscellaneous discharge measurements, Mason County, western Washington,

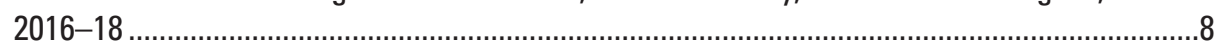

2. Measured monthly groundwater levels, Mason County, western Washington, 2016-18 


\section{Conversion Factors}

U.S. customary units to International System of Units

\begin{tabular}{lcl}
\hline \multicolumn{1}{c}{ Multiply } & By & \multicolumn{1}{c}{ To obtain } \\
\hline inch (in.) & Length & \\
inch (in.) & 2.54 & centimeter $(\mathrm{cm})$ \\
foot (ft) & 25.4 & millimeter $(\mathrm{mm})$ \\
mile (mi) & 0.3048 & meter $(\mathrm{m})$ \\
& 1.609 & kilometer $(\mathrm{km})$ \\
\hline acre & Area & \\
square mile $\left(\mathrm{mi}^{2}\right)$ & 4,047 & square meter $\left(\mathrm{m}^{2}\right)$ \\
\hline & 2.590 & square kilometer $\left(\mathrm{km}^{2}\right)$ \\
\hline cubic foot per second $\left(\mathrm{ft}^{3} / \mathrm{s}\right)$ & Flow rate & \\
\hline
\end{tabular}

Temperature in degrees Celsius $\left({ }^{\circ} \mathrm{C}\right)$ may be converted to degrees Fahrenheit $\left({ }^{\circ} \mathrm{F}\right)$ as follows:

${ }^{\circ} \mathrm{F}=\left(1.8 x^{\circ} \mathrm{C}\right)+32$

\section{Datums}

Vertical coordinate information is referenced to the North American Vertical Datum of 1988 (NAVD 88).

Horizontal coordinate information is referenced to the North American Datum of 1983 (NAD 83).

Altitude, as used in this report, refers to distance above the vertical datum. 


\section{Well-Numbering System}

In Washington, wells are assigned numbers that identify their location in a township, range, section, and 40-acre tract. For example, well number 21N/02W-35D02 indicates that the well is in township 21 north of the Willamette Base Line, and range 2 west of the Willamette Meridian. The numbers immediately following the hyphen indicate the section (35) in the township, and the letter following the section (D) gives the 40 -acre tract of the section. The two-digit sequence number (02) following the letter indicates that the well was the second one inventoried in that 40acre tract. In the illustrations of this report, wells are identified individually using only the section and 40 -acre tract, such as 35D02. The townships and ranges are shown on the map borders.

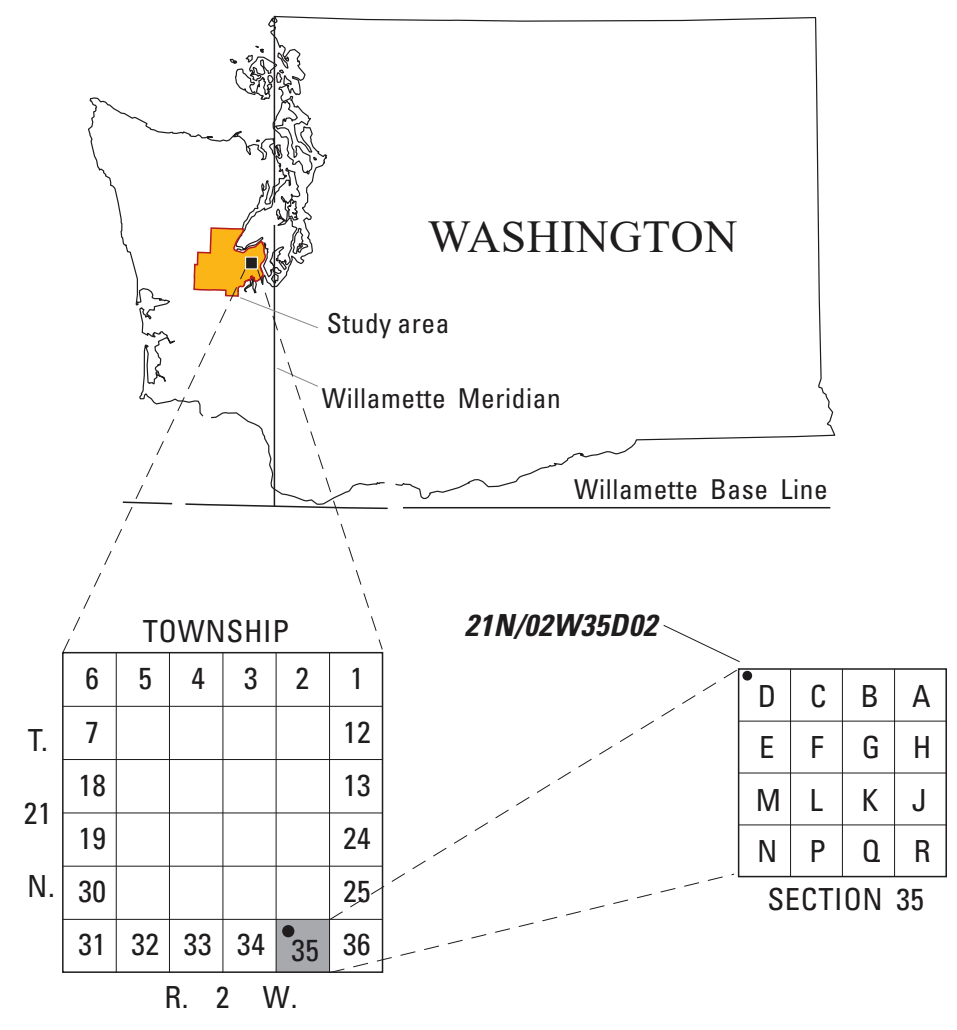





\title{
Groundwater and Surface-Water Data Collection for Mason County, Western Washington, 2016-18
}

\author{
By Alison E. Tecca and Lonna M. Frans
}

\begin{abstract}
Groundwater levels and surface-water flow measurements were collected from August 2016 to September 2018 to provide the Mason Conservation District and other stakeholders with basic knowledge of existing water resources in Mason County, Washington. Additionally, the data were collected with the intent of contributing to informed decision making about groundwater use, management, and conservation throughout the county and for future inclusion in a groundwater model. Data were collected and compiled for 130 sites - 110 wells and 20 miscellaneous surface-water discharge sites. In spring 2016, field reconnaissance was conducted to locate suitable locations for baseflow discharge measurements to be used for estimating groundwater contribution to surface flow. In summer 2016, a field inventory of wells was conducted to acquire locational data and to assess the suitability of the wells for inclusion in a monthly groundwater-level monitoring network. Groundwater levels were measured bimonthly in the 64 wells over 2 years. Streamflow measurements were conducted two times each summer during two summers for each of the 20 surface-water sites.
\end{abstract}

\section{Introduction}

Groundwater is an important resource for domestic, commercial, and industrial use in Mason County, western Washington. In late summer and early autumn, groundwater discharge maintains streamflow for many small creeks and streams throughout the county and for the numerous fish species that inhabit them. Population growth and increases in commercial and industrial activity create a high demand on water resources in the county, yet these resources are insufficiently understood. To quantify groundwater resources and to better understand the potential effects of both natural and anthropogenic influences on groundwater, U.S. Geological Survey (USGS) personnel measured groundwater levels in wells and discharge in streams from August 2016 to September 2018. In the future, these data will be integrated into a numerical groundwater-flow model to be used by water resource managers to simulate potential effects on groundwater and surface-water resources in the study area.

The collection of groundwater data to obtain baseline information substantially helps with efforts to monitor trends in groundwater conditions and to augment critical hydrologic data gaps in the county. Monitoring groundwater levels in conjunction with streamflow is necessary to understand groundwater and surface-water interactions.

\section{Purpose and Scope}

The purpose of this report is to list and describe the results of the groundwater and surface-water data collection effort in Mason County from August 2016 to September 2018. The topics covered in this report include introductory and background information, a description of the methods for making baseflow stream discharge measurements, and a description of the process and methods of the initial field well inventory and subsequent monthly groundwater level measurements.

\section{Study Area}

The project area occupies approximately 720 square miles in Mason County (fig. 1). In the northwestern corner of the project area and partially within Olympic National Forest, Lake Cushman, a 6.2 square mile reservoir, drains into the North Fork Skokomish River. The North and South Fork Skokomish Rivers join the main stem of the Skokomish River to form a broad, alluvium-filled valley about 9 miles from its outlet into Hood Canal. The northern boundary of the project area extends along the southern arm of Hood Canal east towards Belfair and south following the Mason County line to Totten Inlet and Oyster Bay. In the southwestern part of the county, major tributaries of the Satsop River, which flows south, originate in upland prairies and the southern foothills of the Olympic Mountains. Wetlands and springs are prominent in the study area and contribute to stream baseflow conditions in the summer months. 


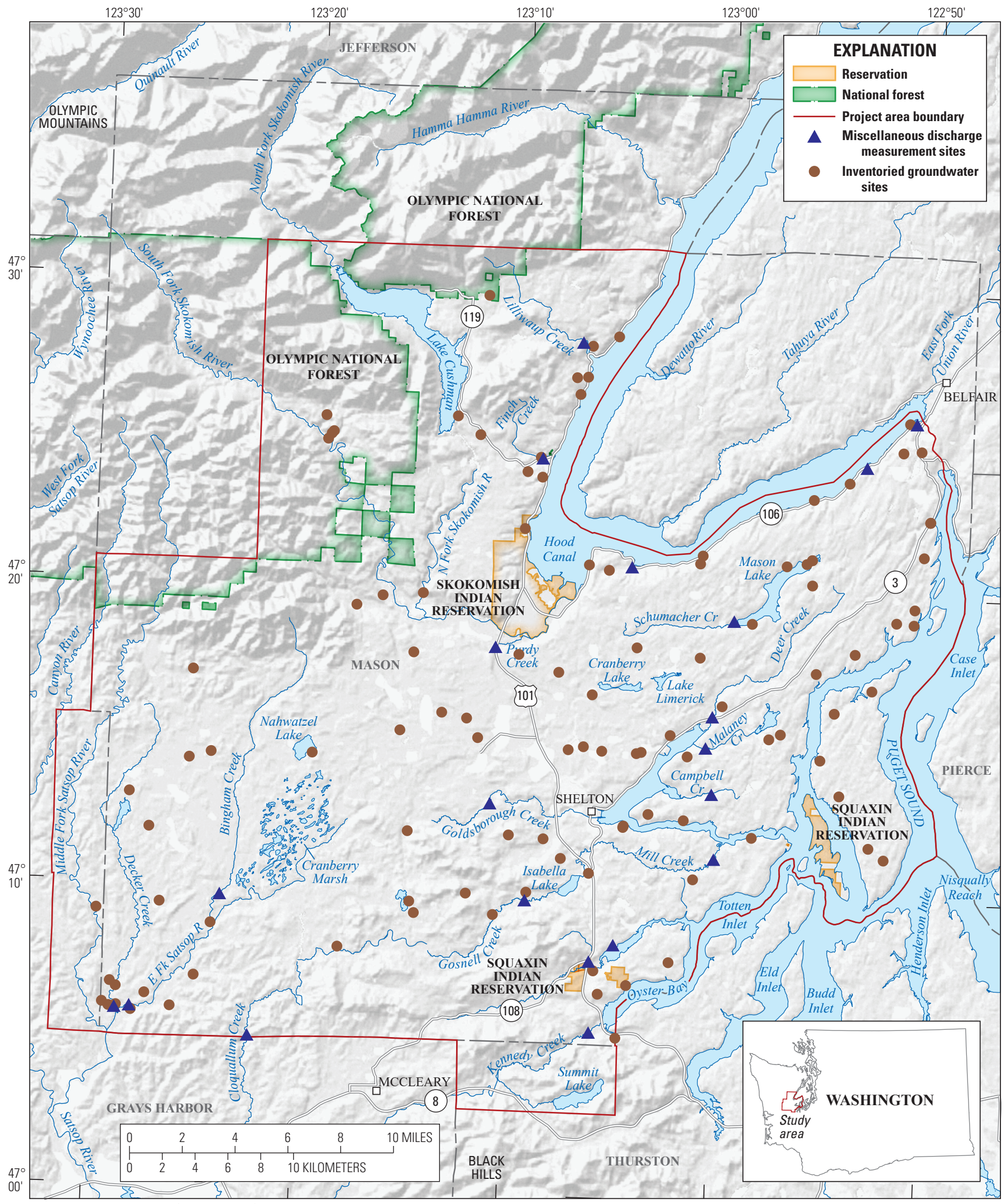

Base map modified from Esri (copyright 2018), U.S.Geological Survey, and other digital data. Lambert

Conformal Conic projection: State Plane Washington South. North American Datum of 1983.

Figure 1. General project study area, Mason County, western Washington, 2016-18. 
A major part of the study area is underlain by a northeast thickening sequence of unconsolidated Quaternary glacial and interglacial surface deposits derived from both alpine and continental glaciations. Most of these deposits contain water-bearing aquifers. Bedrock units are comprised of Tertiary basalts of the Crescent Formation with interbeds of undifferentiated marine sedimentary rock. These bedrock units are exposed in the Olympic Mountains and as outcrops to the south and southwest of the city of Shelton in the Black Hills. Due to their general impermeability, Tertiary deposits in the county contain no significant aquifers (Molenaar and Noble, 1970).

The study area has a temperate marine climate characterized by mild, wet winters and warm, dry summers, with temperature and rainfall values moderated by the Pacific Ocean and Puget Sound. Groundwater recharge is driven largely by precipitation, which averages 86.9 inches per year in the county based on 30-year normals (PRISM Climate Group, 2018).

The population of the project area is approximately 52,000 people, with about 20 percent living within the city of Shelton (Washington State Office of Financial Management, 2015).

\section{Methods}

\section{Surface-Water Measurement Methods}

Synoptic streamflow measurements were collected during baseflow conditions at 20 non-gaged locations in August and September 2016 and again in July and August 2017 to augment streamflow data from 6 active USGS streamgages in the study area (fig. 2). These data were used to quantify the amount of surface water leaving the study area and to determine baseflow amounts during low-flow conditions.

Streamflow measurements were made by USGS personnel using a FlowTracker ${ }^{\circledR}$ handheld acoustic Doppler velocimeter (ADV). When streams were too small for conditions to permit the use of an ADV, a 6-inch modified Parshall flume according to standard techniques of the USGS (Rantz, 1982). All streams were shallow and easily wadable by field personnel during the course of the measurements. Streamflow measurement data are presented in table 1 (at back of report) and stored in the USGS National Water Information System (NWIS).

The USGS assigns accuracy ratings of good, fair, or poor to streamflow measurements based on the equipment, character of the measurement section, number of observations, stability of stage, wind conditions, and the accuracy of depth and velocity measurements (Rantz, 1982, p. 179). "Good" indicates that the measurements are judged to be within 5 percent of true values, "fair" indicates that the measurements are judged to be within 8 percent of true values, and "poor" indicates that the measurements are judged not to be within 8 percent of true values.

\section{Groundwater Measurement Methods}

Available well records were compiled, and sites were located in the field during summer 2016. Well selection was generally based on the availability of a drillers' report for the well (obtained from the Washington Department of Ecology or other sources), the availability of lithologic and construction information on the drillers' report, and permission from the owner or tenant to repeatedly visit the site. At the time of inventory, the groundwater level was measured, if accessible, and a field form was completed noting the location from GPS units, well construction information, and water level at each site. The depth to water in wells was measured following USGS procedures outlined in the USGS Techniques and Method report 1-A1 "Groundwater Technical Procedures of the U.S. Geological Survey" (Cunningham and Schalk, comps., 2011). This report provides detailed, illustrated instructions for the implementation of common field methods for collecting groundwater data.

Global Positioning System (GPS) hand-held units were used during the inventory to determine the latitude and longitude of each site. The locations of wells as determined by GPS were documented with latitude and longitude values reported in ddmmss.ss (degrees, minutes, decimal seconds). The latitude and longitude were read and recorded on the field sheet after water-level measurements were completed to give sufficient time for GPS readings to stabilize. Values between the GPS unit and those recorded on the field sheet were double-checked before departing the site. A sketch map showing location of the well was made on the field sheet. This map is intended for future visits and can include measured distances (vehicle odometer) from road intersections and estimated or paced distances from identifiable site features (such as, buildings, driveways, creeks, or fences).

Land-surface altitudes for each well were obtained from a digital elevation model with 10 -square-meter cells using the latitude and longitude for each site. Information for all wells was entered in the USGS National Water Information System database (https://waterdata.usgs.gov/).

Water levels in wells were measured with steel or electronic tapes. All measurements were confirmed-taken twice, with at least 3 minutes between measurements. The confirmation measurement needed to be within 0.02 feet to be considered a static water-level measurement. If the initial measurements were not in agreement, additional measurements were made until the water level status was determined (for example, recovering or pumping). 


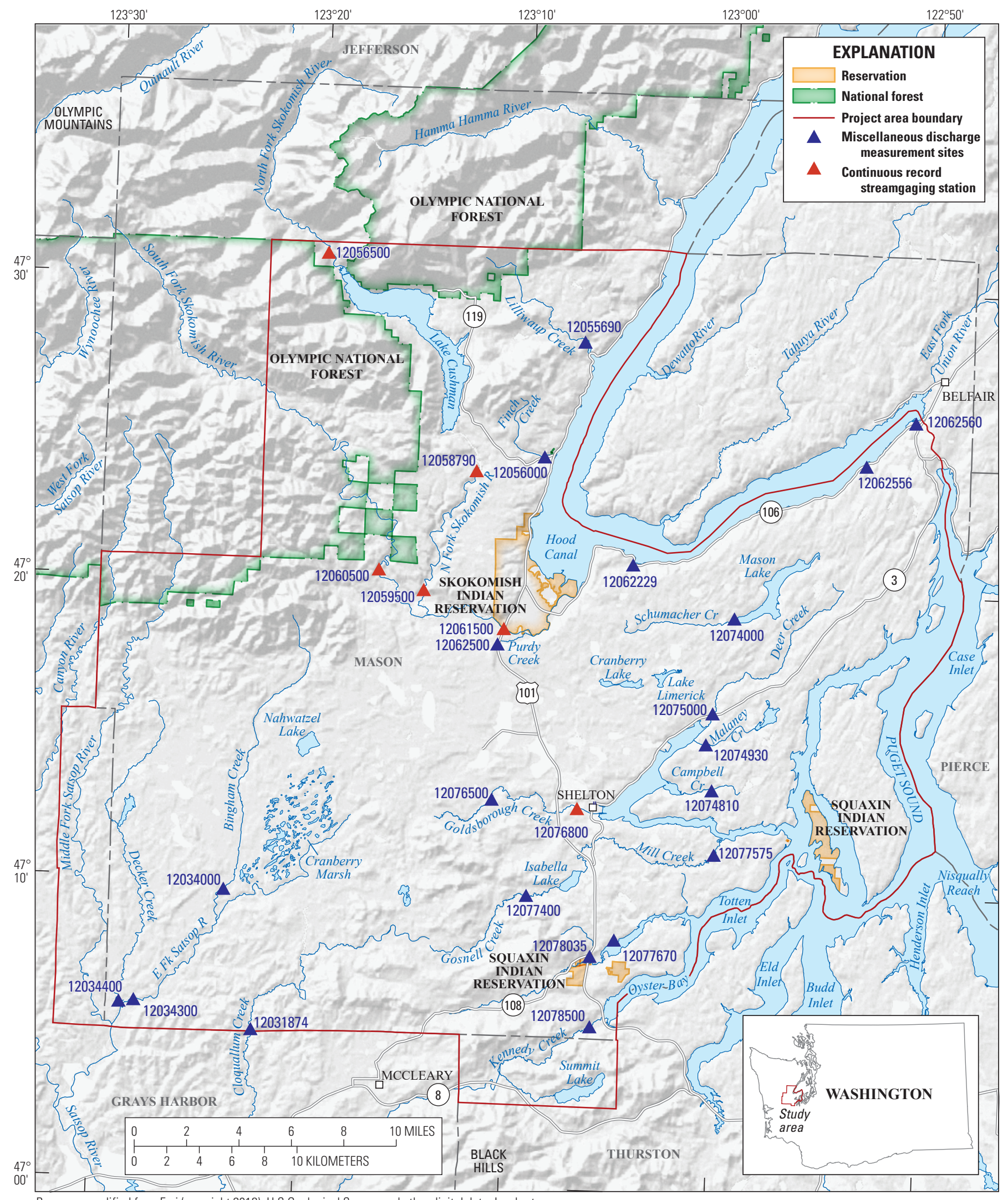

Base map modified from Esri (copyright 2018), U.S.Geological Survey, and other digital data. Lambert

Conformal Conic projection: State Plane Washington South. North American Datum of 1983.

Figure 2. Surface-water site locations, Mason County, western Washington, 2016-18. 
Of the roughly 110 wells inventoried, 64 wells with measurable water levels were selected for a monthly groundwater-monitoring network run by USGS personnel. The project wells were monitored bi-monthly, with approximately one half of the wells visited each month and the other half visited the next month. All hydrologic data collected during this study was compiled and entered into the USGS National Water Information System (NWIS) database (https:// waterdata.usgs.gov/).

\section{Results}

The primary components of this investigation included a field inventory of wells, establishing a monthly groundwaterlevel monitoring network and measuring stream discharge under baseflow conditions.

\section{Streamflow Measurements}

Synoptic stream baseflow discharge measurements were collected at 20 non-gaged locations in August and September 2016 and again in July and August 2017 to augment streamflow data from 6 active USGS gaging stations currently operating in the study area (fig. 2). These data were used to quantify the amount of baseflow in streams (table 1).

A large variety of sites were measured with measured discharge ranging from 0.01 cubic feet per second at the Little Skookum inlet tributary (Little Skookum Inlet Trib nr mouth nr Kamilche, WA; U.S. Geological Survey site 12077670) to
167 cubic feet per second in the East Fork Satsop River (EF Satsop River at Shafer St Park nr Satsop, WA; U.S. Geological Survey site 12034300). Discharge values were generally higher in 2017 than in 2016 for the same sites.

\section{Groundwater Network}

In summer 2016, a field inventory was conducted to locate potential sites and to acquire site data, including measuring depth to water in as many wells as possible. The location and associated information for wells in the county were obtained from past reports and the Washington Department of Ecology. The intent of the field inventory was to collect data from wells evenly distributed throughout the county. This was not possible in all areas because of lack of development of some areas, or lack of permission to access some sites. Locations of the inventoried wells are shown in figure 1.

After completion of the well inventory, 64 of the fieldlocated wells were selected for use in a monthly groundwaterlevel monitoring network. Starting in August 2016, the USGS established a monthly network, and USGS personnel measured the wells through September 2018 (table 2 [at back of report] and fig. 3).

The monthly monitoring wells ranged in depth from 35 to 678 feet below land surface. The depths to water ranged from about 298 feet below land surface to 0.3 feet above land surface. Hydrographs are presented for wells 22N/05W-09G02 and 20N/03W-04Q04D1 as examples of some of the variation in water levels that were collected (fig. 4). 


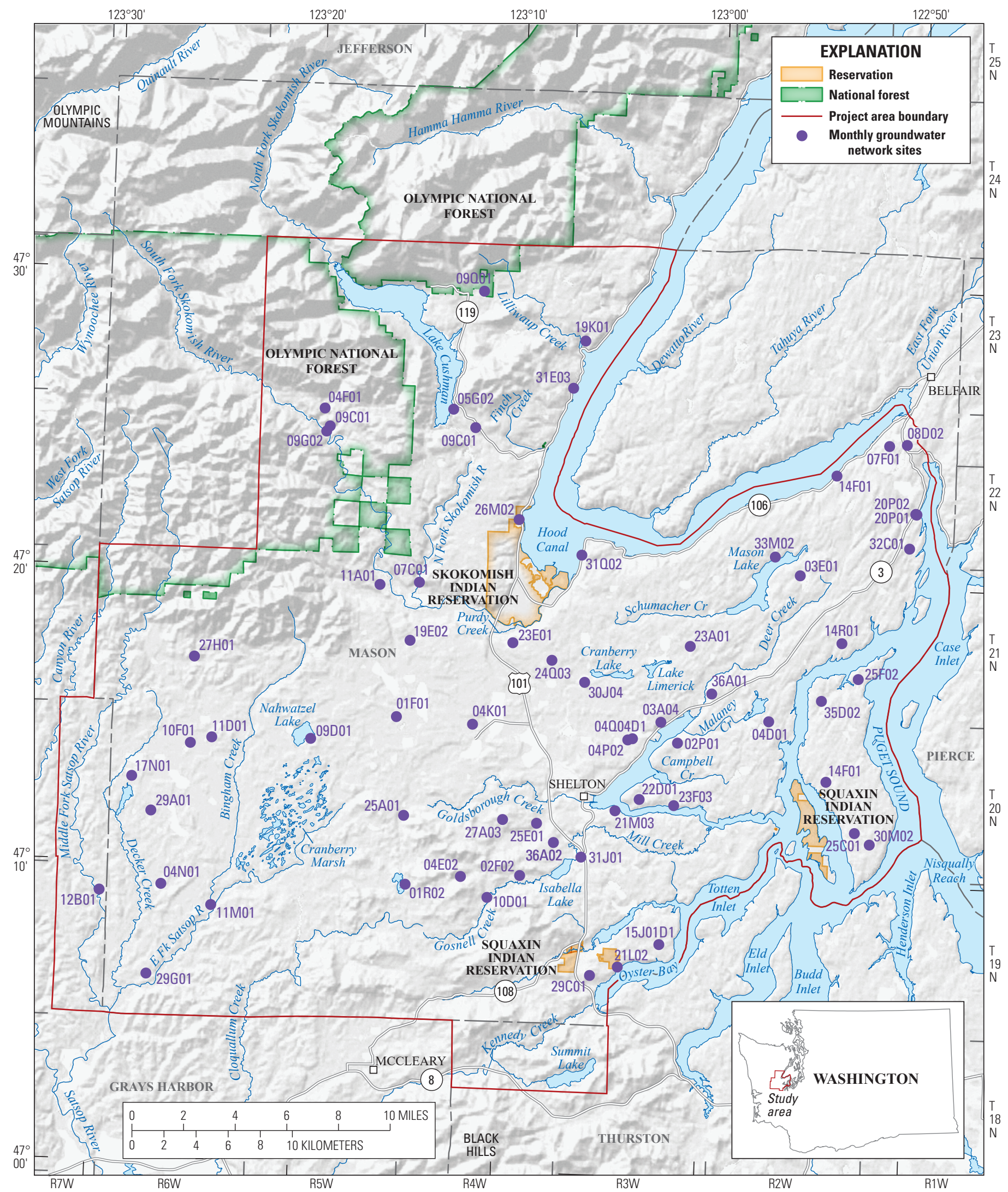

Base map modified from Esri (copyright 2018), U.S.Geological Survey, and other digital4data. Lambert Conformal Conic projection: State Plane Washington South. North American Datum of 1983.

Figure 3. Groundwater site locations, Mason County, western Washington, 2016-18. 


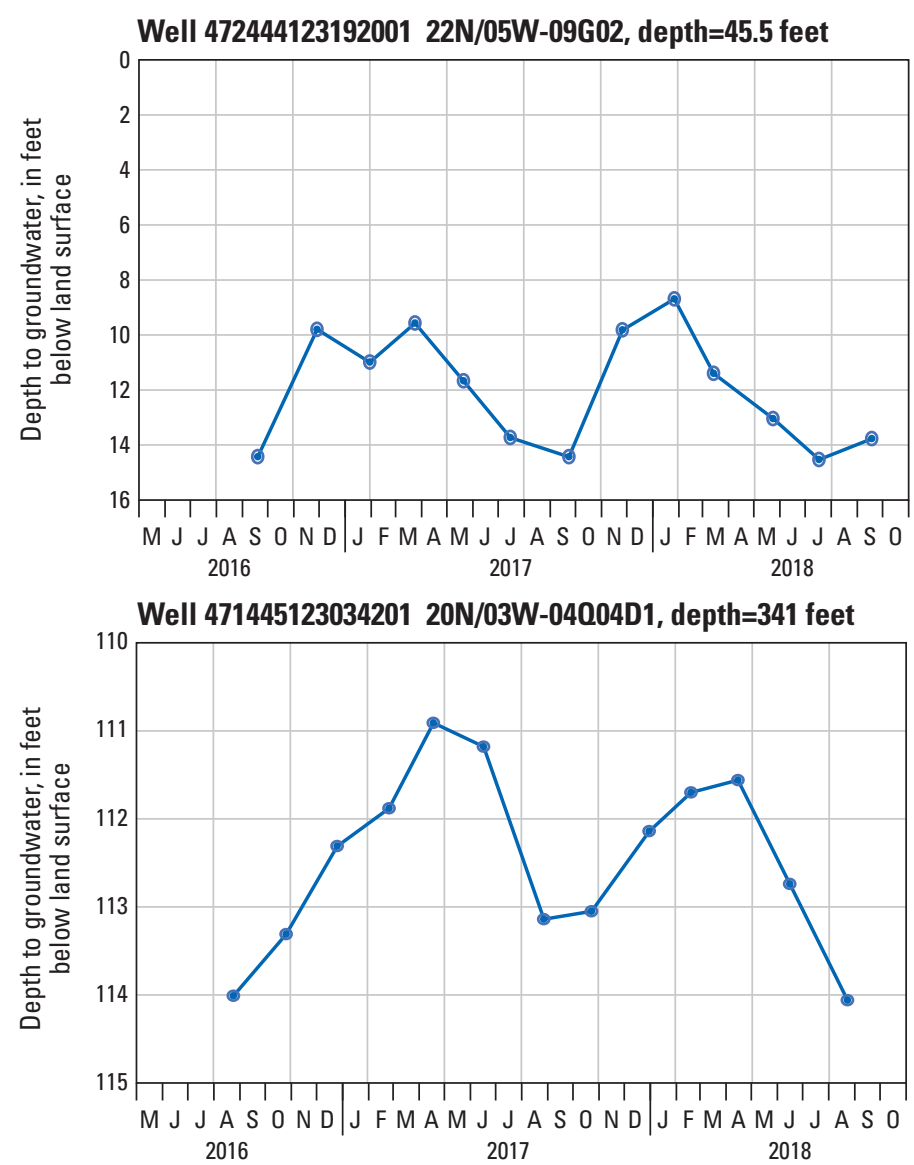

Figure 4. Examples of hydrographs for monthly waterlevel wells, Mason County, western Washington, 2016-18.

\section{Summary}

Groundwater and surface-water data were collected from August 2016 to September 2018 to provide the Mason Conservation District and other stakeholders basic knowledge of existing water resources in Mason County, Washington. Additionally, the data were collected with the intent of contributing to informed decision making about groundwater use, management, and conservation throughout the county. Data were collected and compiled for 130 total sites-110 wells and 20 surface-water sites. In spring 2016, a field reconnaissance was done to locate suitable locations for baseflow discharge measurements to be used for estimating groundwater contribution to surface flow. In summer 2016 a field inventory of wells was conducted to acquire locational data and to assess the suitability of the wells for inclusion in a monthly groundwater-level monitoring network. Water levels were measured bimonthly in the 64 wells over two years. Streamflow measurements were made 2 times each summer over 2 summers for each of the 20 streams.

\section{Acknowledgments}

The USGS gratefully acknowledges the Mason County landowners who allowed access to their lands and wells and who shared their knowledge about the water resources of the area.

\section{References Cited}

Cunningham, W.L., and Schalk, C.W., comps., 2011, Groundwater technical procedures of the U.S. Geological Survey: U.S. Geological Survey Techniques and Methods 1-A1, 151 p. [Also available at https://pubs.usgs.gov/ $\mathrm{tm} / 1 \mathrm{a} 1 /]$

Molenaar, Dee, and Noble, J.B., 1970, Geology and related ground-water occurrence, southeastern Mason County, Washington: Washington Department of Water Resources Water Survey Bulletin no. 29, 145 p.

PRISM Climate Group, 2018, PRISM climate data: Oregon State University, accessed September 2018 at http://prism. oregonstate.edu.

Rantz, S.E., 1982, Measurement and computation of streamflow: U.S. Geological Survey Water Supply Paper 2175, 2 v., 631 p., https://pubs.usgs.gov/wsp/wsp2175/.

Washington State Office of Financial Management, 2015, Washington data \& research - Population and demographics - GIS data: Washington State Office of Financial Management, accessed 2015 at http://www.ofm. wa.gov/pop/geographic/default.asp. 
Table 1. Miscellaneous discharge measurements, Mason County, western Washington, 2016-18.

[Latitude and Longitude: Latitude and longitude at site in decimal degrees referenced to North American Datum of 1983. Abbreviations: $\mathrm{ft}^{3} / \mathrm{s}$, cubic foot per second; ft, foot; $\mathrm{ft} / \mathrm{s}$, foot per second; $\mathrm{ft}^{2}$, square foot; -, no data]

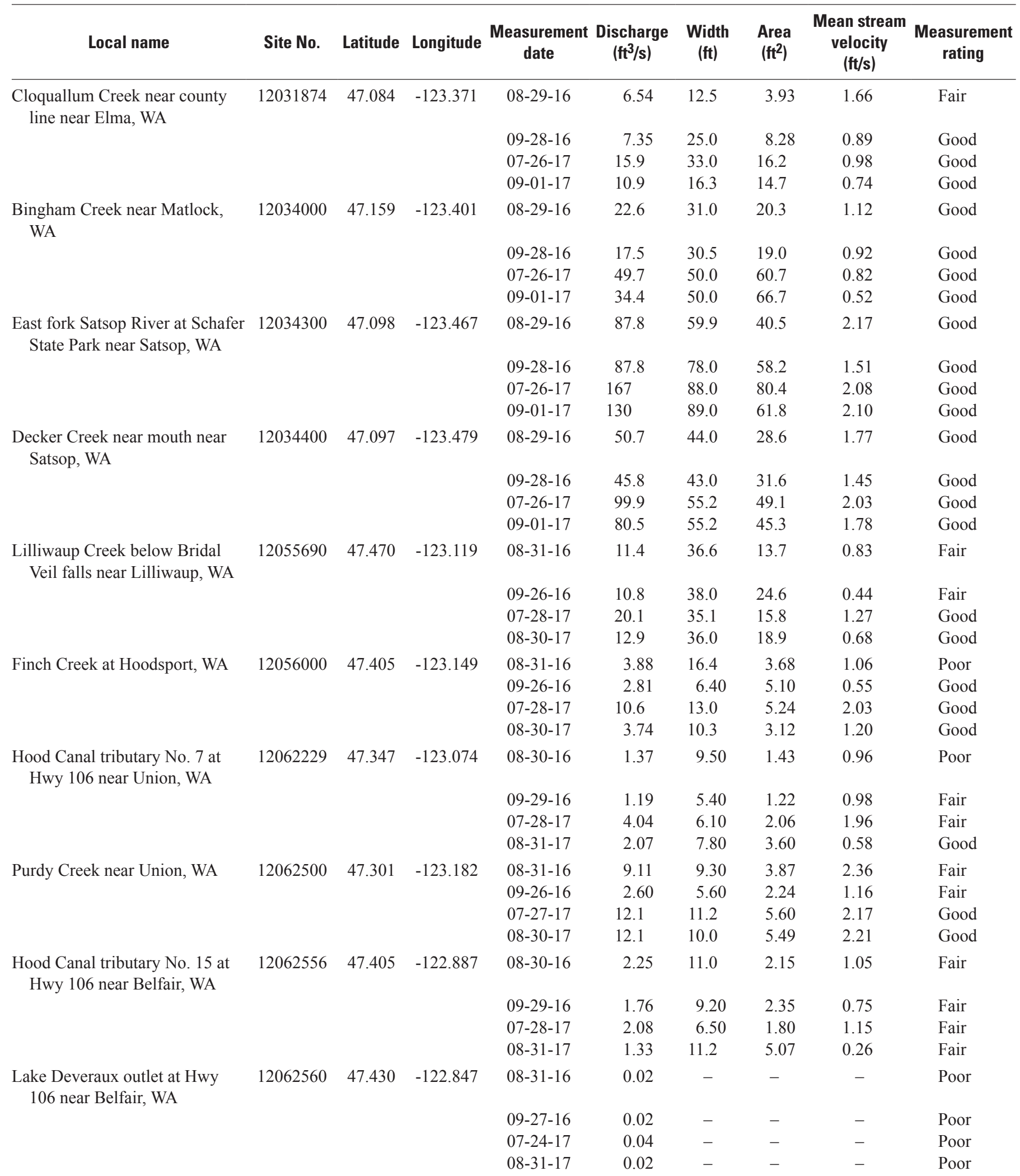


Table 1. Miscellaneous discharge measurements, Mason County, western Washington, 2016-18.-Continued

\begin{tabular}{|c|c|c|c|c|c|c|c|c|c|}
\hline Local name & Site No. & Latitude & Longitude & $\begin{array}{l}\text { Measurement } \\
\quad \text { date }\end{array}$ & $\begin{array}{l}\text { Discharge } \\
\left(\mathrm{ft}^{3} / \mathrm{s}\right)\end{array}$ & $\begin{array}{l}\text { Width } \\
\text { (ft) }\end{array}$ & $\begin{array}{c}\text { Area } \\
\left(\mathrm{ft}^{2}\right)\end{array}$ & $\begin{array}{l}\text { Mean stream } \\
\text { velocity } \\
\text { (ft/s) }\end{array}$ & $\begin{array}{l}\text { Measurement } \\
\quad \text { rating }\end{array}$ \\
\hline \multirow[t]{4}{*}{$\begin{array}{l}\text { Shumocher Creek (headwaters of } \\
\text { Sherwood Creek) near Union, } \\
\text { WA }\end{array}$} & 12074000 & 47.319 & -122.990 & 08-30-16 & 8.66 & 18.2 & 7.41 & 1.17 & Good \\
\hline & & & & $09-27-16$ & 6.91 & 18.5 & 7.63 & 0.91 & Good \\
\hline & & & & $07-28-17$ & 14.1 & 19.6 & 13.9 & 1.02 & Good \\
\hline & & & & $08-31-17$ & 11.1 & 20.3 & 12.6 & 0.88 & Good \\
\hline \multirow[t]{4}{*}{ Campbell Creek near Agate, WA } & 12074810 & 47.224 & -123.004 & 08-30-16 & 1.20 & 6.50 & - & - & Poor \\
\hline & & & & $09-27-16$ & 0.90 & 7.50 & 4.30 & 0.21 & Good \\
\hline & & & & $07-25-17$ & 1.71 & 6.30 & 2.28 & 0.75 & Good \\
\hline & & & & $08-28-17$ & 1.10 & 6.20 & 2.14 & 0.52 & Good \\
\hline \multirow{4}{*}{$\begin{array}{l}\text { Malaney Creek at Agate Road } \\
\text { near Agate, WA }\end{array}$} & 12074930 & 47.249 & -123.010 & $08-30-16$ & 0.59 & 5.60 & 0.80 & 0.74 & Poor \\
\hline & & & & $09-27-16$ & 0.34 & - & - & - & Poor \\
\hline & & & & $07-25-17$ & 1.57 & 4.70 & 1.28 & 1.22 & Fair \\
\hline & & & & $08-28-17$ & 0.98 & 4.30 & 1.17 & 0.83 & Fair \\
\hline \multirow[t]{4}{*}{ Deer Creek near Shelton, WA } & 12075000 & 47.267 & -123.005 & $08-30-16$ & 21.1 & 20.0 & 15.2 & 1.39 & Fair \\
\hline & & & & $09-27-16$ & 21.3 & 19.0 & 15.4 & 1.38 & Good \\
\hline & & & & $07-25-17$ & 25.7 & 26.3 & 39.0 & 0.66 & Poor \\
\hline & & & & $08-28-17$ & 21.7 & 28.4 & 41.6 & 0.52 & Poor \\
\hline \multirow{4}{*}{$\begin{array}{l}\text { Goldsborough Creek near } \\
\text { Shelton, WA }\end{array}$} & 12076500 & 47.215 & -123.182 & $08-29-16$ & 21.6 & 29.6 & 12.6 & 1.72 & Poor \\
\hline & & & & $09-26-16$ & 21.3 & 31.0 & 32.0 & 0.66 & Fair \\
\hline & & & & $07-27-17$ & 28.5 & 33.8 & 36.6 & 0.78 & Fair \\
\hline & & & & $08-29-17$ & 22.8 & 33.3 & 33.5 & 0.68 & Good \\
\hline \multirow{4}{*}{$\begin{array}{l}\text { Gosnell Creek at West Bolling } \\
\text { Road near Shelton, WA }\end{array}$} & 12077395 & 47.157 & -123.170 & 08-31-16 & 9.48 & 12.9 & - & - & Good \\
\hline & & & & $09-28-16$ & 9.65 & 11.5 & 5.35 & 1.81 & Good \\
\hline & & & & $07-27-17$ & 15.7 & 15.6 & 9.67 & 1.63 & Good \\
\hline & & & & $08-29-17$ & 13.2 & 15.1 & 8.30 & 1.59 & Good \\
\hline \multirow{4}{*}{$\begin{array}{l}\text { Mill Creek at Arcadia Road near } \\
\text { Shelton, WA }\end{array}$} & 12077575 & 47.188 & -123.001 & $08-30-16$ & 5.81 & 22.9 & 7.98 & 0.73 & Poor \\
\hline & & & & $09-27-16$ & 12.3 & 26.5 & 17.5 & 0.70 & Poor \\
\hline & & & & $07-27-17$ & 21.2 & 26.4 & 15.2 & 1.39 & Good \\
\hline & & & & $08-29-17$ & 16.8 & 24.3 & 12.2 & 1.38 & Good \\
\hline \multirow{4}{*}{$\begin{array}{l}\text { Little Skookum Inlet tributary } \\
\text { near mouth near Kamilche, } \\
\text { WA }\end{array}$} & 12077670 & 47.140 & -123.080 & $08-26-16$ & 0.04 & - & - & - & Poor \\
\hline & & & & $09-26-16$ & 0.01 & - & - & - & Poor \\
\hline & & & & $07-24-17$ & 0.69 & - & - & - & Poor \\
\hline & & & & $08-30-17$ & 0.04 & - & - & - & Poor \\
\hline \multirow{4}{*}{$\begin{array}{l}\text { Little Skookum Creek at Hwy } \\
108 \text { at Kamilche, WA }\end{array}$} & 12078035 & 47.130 & -123.099 & $08-29-16$ & 0.14 & 5.60 & 1.30 & 0.11 & Poor \\
\hline & & & & $09-28-16$ & 0.02 & - & - & - & Poor \\
\hline & & & & $07-27-17$ & 0.65 & 6.40 & 2.09 & 0.31 & Fair \\
\hline & & & & $08-29-17$ & 0.60 & 7.10 & 2.36 & 0.25 & Fair \\
\hline \multirow{4}{*}{$\begin{array}{l}\text { Kennedy Creek near New } \\
\text { Kamilche, WA }\end{array}$} & 12078500 & 47.092 & -123.097 & $08-29-16$ & 3.55 & 14.3 & 4.19 & 0.85 & Poor \\
\hline & & & & $09-26-16$ & 3.96 & 13.3 & 5.14 & 0.77 & Poor \\
\hline & & & & $07-26-17$ & 8.88 & 6.71 & 3.34 & 2.66 & Good \\
\hline & & & & $08-28-17$ & 6.47 & 6.00 & 2.78 & 2.33 & Fair \\
\hline
\end{tabular}


Table 2. Measured monthly groundwater levels, Mason County, western Washington, 2016-18.

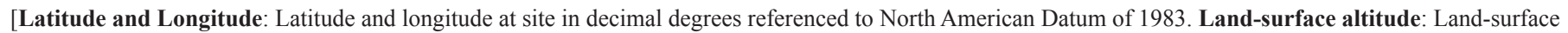
altitude at site in feet above North American Vertical Datum of 1988 . Groundwater level status: G, nearby flowing; P, pumping; R, recently pumped; S, nearby

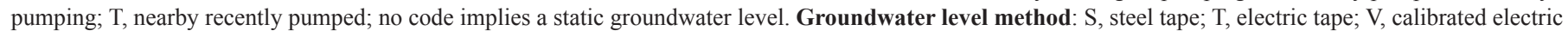
tape. Abbreviation: ft, foot]

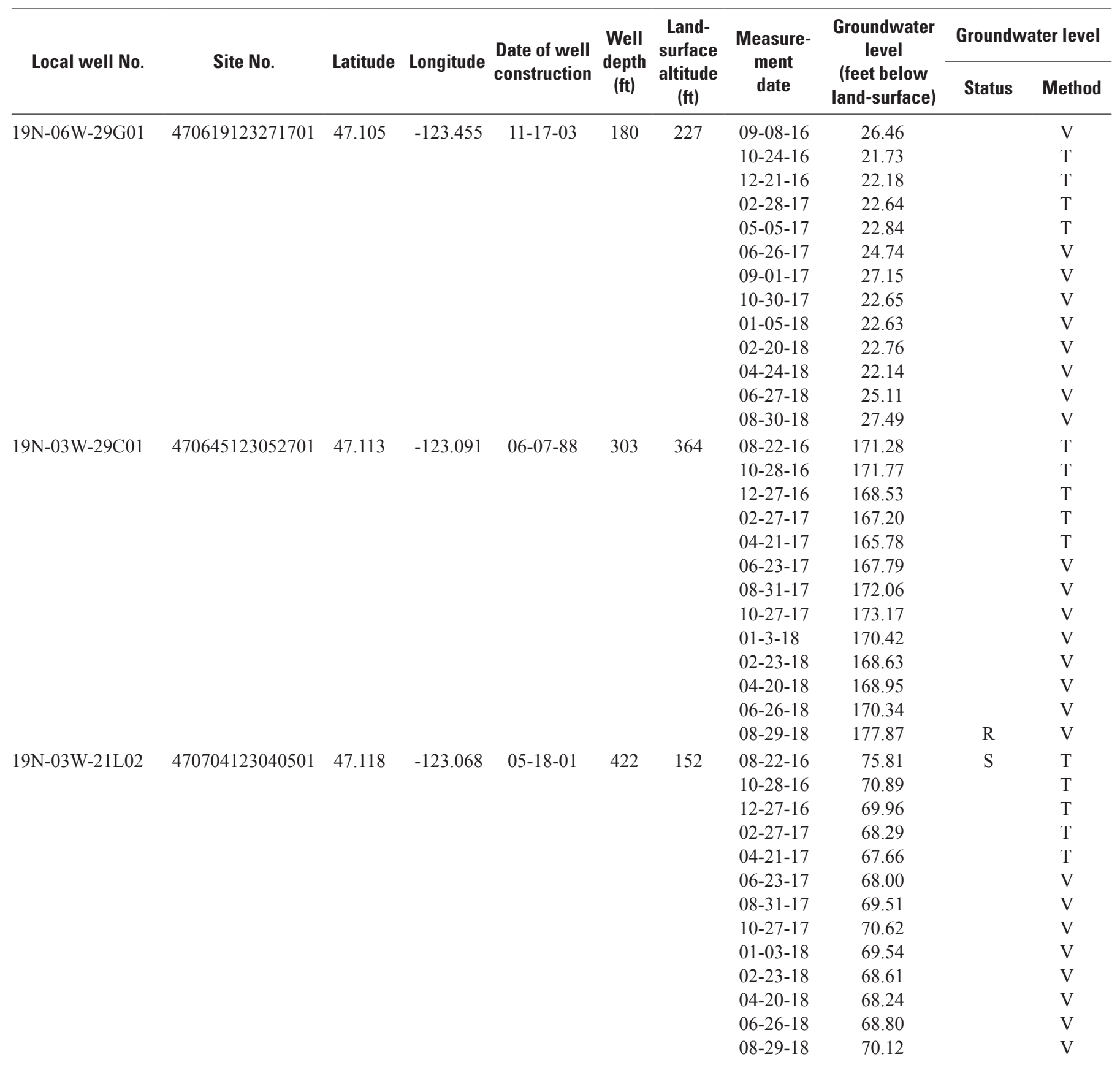


Table 211

Table 2. Measured monthly groundwater levels, Mason County, western Washington, 2016-18.-Continued

\begin{tabular}{|c|c|c|c|c|c|c|c|c|c|c|}
\hline \multirow{2}{*}{ Local well No. } & \multirow{2}{*}{ Site No. } & \multirow{2}{*}{ Latitude } & \multirow{2}{*}{ Longitude } & \multirow{2}{*}{$\begin{array}{l}\text { Date of well } \\
\text { construction }\end{array}$} & \multirow{2}{*}{$\begin{array}{l}\text { Well } \\
\text { depth } \\
\text { (ft) }\end{array}$} & \multirow{2}{*}{$\begin{array}{l}\text { Land- } \\
\text { surface } \\
\text { altitude } \\
\text { (ft) }\end{array}$} & \multirow{2}{*}{$\begin{array}{c}\text { Measure- } \\
\text { ment } \\
\text { date }\end{array}$} & \multirow{2}{*}{$\begin{array}{c}\text { Groundwater } \\
\text { level } \\
\text { (feet below } \\
\text { land-surface) }\end{array}$} & \multicolumn{2}{|c|}{ Groundwater level } \\
\hline & & & & & & & & & Status & Method \\
\hline \multirow[t]{13}{*}{ 19N-03W-15J01D1 } & 470752123020501 & 47.131 & -123.035 & $07-24-93$ & 134.6 & 169 & $08-22-16$ & 105.40 & & $\mathrm{~T}$ \\
\hline & & & & & & & $10-28-16$ & 105.28 & & $\mathrm{~T}$ \\
\hline & & & & & & & $12-27-16$ & 105.40 & & $\mathrm{~T}$ \\
\hline & & & & & & & $02-27-17$ & 104.92 & & $\mathrm{~T}$ \\
\hline & & & & & & & $04-21-17$ & 105.30 & & $\mathrm{~T}$ \\
\hline & & & & & & & $06-23-17$ & 105.00 & & $\mathrm{~V}$ \\
\hline & & & & & & & $08-31-17$ & 105.06 & & $\mathrm{~V}$ \\
\hline & & & & & & & $10-27-17$ & 105.13 & & $\mathrm{~V}$ \\
\hline & & & & & & & 01-03-18 & 105.01 & & $\mathrm{~V}$ \\
\hline & & & & & & & $02-23-18$ & 104.87 & & $\mathrm{~V}$ \\
\hline & & & & & & & $04-20-18$ & 104.40 & & $\mathrm{~V}$ \\
\hline & & & & & & & $06-26-18$ & 104.23 & & $\mathrm{~V}$ \\
\hline & & & & & & & 08-29-18 & 103.88 & & $\mathrm{~V}$ \\
\hline \multirow[t]{13}{*}{ 19N-06W-11M01 } & 470842123241301 & 47.145 & -123.404 & $11-17-99$ & 50 & 234 & 09-08-16 & 6.33 & & $\mathrm{~V}$ \\
\hline & & & & & & & $10-24-16$ & 4.41 & & $\mathrm{~T}$ \\
\hline & & & & & & & $12-21-16$ & 3.46 & & $\mathrm{~T}$ \\
\hline & & & & & & & $02-28-17$ & 3.81 & & $\mathrm{~T}$ \\
\hline & & & & & & & $05-05-17$ & 3.80 & & $\mathrm{~T}$ \\
\hline & & & & & & & $06-26-17$ & 5.21 & & $\mathrm{~V}$ \\
\hline & & & & & & & 09-01-17 & 6.11 & & $\mathrm{~V}$ \\
\hline & & & & & & & $10-30-17$ & 6.10 & & $\mathrm{~V}$ \\
\hline & & & & & & & 01-05-18 & 3.49 & & $\mathrm{~V}$ \\
\hline & & & & & & & $02-20-18$ & 3.68 & & $\mathrm{~V}$ \\
\hline & & & & & & & $04-24-18$ & 3.54 & & V \\
\hline & & & & & & & $06-27-18$ & 5.74 & & $\mathrm{~V}$ \\
\hline & & & & & & & 08-30-18 & 6.29 & & $\mathrm{~V}$ \\
\hline \multirow[t]{13}{*}{ 19N-07W-12B01 } & 470904123294401 & 47.151 & -123.496 & $05-08-07$ & 56 & 204 & $08-25-16$ & 23.86 & & $\mathrm{~S}$ \\
\hline & & & & & & & $10-24-16$ & 21.21 & & $\mathrm{~T}$ \\
\hline & & & & & & & $12-21-16$ & 21.27 & & $\mathrm{~T}$ \\
\hline & & & & & & & $02-28-17$ & 22.18 & & $\mathrm{~S}$ \\
\hline & & & & & & & $05-05-17$ & 22.28 & & $\mathrm{~S}$ \\
\hline & & & & & & & $06-26-17$ & 23.15 & & $\mathrm{~S}$ \\
\hline & & & & & & & 09-01-17 & 23.77 & & $\mathrm{~S}$ \\
\hline & & & & & & & $10-30-17$ & 23.12 & & $\mathrm{~S}$ \\
\hline & & & & & & & 01-05-18 & 21.66 & & $\mathrm{~S}$ \\
\hline & & & & & & & $02-20-18$ & 22.10 & & V \\
\hline & & & & & & & 04-24-18 & 22.24 & & $\mathrm{~S}$ \\
\hline & & & & & & & $06-27-18$ & 24.06 & & $\mathrm{~S}$ \\
\hline & & & & & & & 08-30-18 & 24.22 & & $\mathrm{~S}$ \\
\hline \multirow[t]{13}{*}{ 19N-04W-10D01 } & 470916123103701 & 47.154 & -123.177 & 07-05-94 & 97 & 167 & $08-25-16$ & 8.53 & $\mathrm{R}$ & $\mathrm{T}$ \\
\hline & & & & & & & $10-27-16$ & 3.67 & & $\mathrm{~T}$ \\
\hline & & & & & & & $12-22-16$ & 2.38 & & $\mathrm{~T}$ \\
\hline & & & & & & & $02-24-17$ & 2.11 & & $\mathrm{~T}$ \\
\hline & & & & & & & $04-24-17$ & 2.39 & & $\mathrm{~T}$ \\
\hline & & & & & & & $07-03-17$ & 4.71 & & $\mathrm{~V}$ \\
\hline & & & & & & & 09-01-17 & 6.72 & & $\mathrm{~V}$ \\
\hline & & & & & & & $10-31-17$ & 5.94 & & $\mathrm{~V}$ \\
\hline & & & & & & & 01-03-18 & 2.22 & & $\mathrm{~V}$ \\
\hline & & & & & & & $02-22-18$ & 2.91 & & $\mathrm{~V}$ \\
\hline & & & & & & & 04-19-18 & 2.12 & & $\mathrm{~V}$ \\
\hline & & & & & & & $06-28-18$ & 5.59 & & $\mathrm{~V}$ \\
\hline & & & & & & & 08-24-18 & 8.47 & $\mathrm{P}$ & $\mathrm{V}$ \\
\hline
\end{tabular}




\section{Groundwater and Surface-Water Data Collection for Mason County, Western Washington, 2016-18}

Table 2. Measured monthly groundwater levels, Mason County, western Washington, 2016-18.-Continued

\begin{tabular}{|c|c|c|c|c|c|c|c|c|c|c|}
\hline \multirow{2}{*}{ Local well No. } & \multirow{2}{*}{ Site No. } & \multirow{2}{*}{ Latitude } & \multirow{2}{*}{ Longitude } & \multirow{2}{*}{$\begin{array}{l}\text { Date of well } \\
\text { construction }\end{array}$} & \multirow{2}{*}{$\begin{array}{c}\text { Well } \\
\text { depth } \\
\text { (ft) }\end{array}$} & \multirow{2}{*}{$\begin{array}{l}\text { Land- } \\
\text { surface } \\
\text { altitude } \\
\text { (ft) }\end{array}$} & \multirow{2}{*}{$\begin{array}{l}\text { Measure- } \\
\text { ment } \\
\text { date }\end{array}$} & \multirow{2}{*}{$\begin{array}{c}\text { Groundwater } \\
\text { level } \\
\text { (feet below } \\
\text { land-surface) }\end{array}$} & \multicolumn{2}{|c|}{ Groundwater level } \\
\hline & & & & & & & & & Status & Method \\
\hline \multirow{8}{*}{ 19N-06W-04N01 } & 470921123264301 & 47.156 & -123.445 & 07-09-99 & 61 & 295 & $08-05-16$ & 35.57 & & $\mathrm{~V}$ \\
\hline & & & & & & & $08-23-16$ & 42.02 & $\mathrm{P}$ & $\mathrm{T}$ \\
\hline & & & & & & & $10-24-16$ & 19.12 & & $\mathrm{~T}$ \\
\hline & & & & & & & $05-05-17$ & 23.02 & & $\mathrm{~T}$ \\
\hline & & & & & & & $06-26-17$ & 30.76 & $\mathrm{R}$ & $\mathrm{V}$ \\
\hline & & & & & & & $09-01-17$ & 33.90 & $\mathrm{R}$ & $\mathrm{V}$ \\
\hline & & & & & & & $10-30-17$ & 34.41 & & $\mathrm{~V}$ \\
\hline & & & & & & & $01-05-18$ & 19.01 & & $\mathrm{~V}$ \\
\hline \multirow[t]{11}{*}{ 19N-05W-01R02 } & 470937123144101 & 47.160 & -123.245 & $06-03-95$ & 55 & 513 & $08-24-16$ & 54.67 & $\mathrm{P}$ & $\mathrm{T}$ \\
\hline & & & & & & & $10-27-16$ & 26.54 & & $\mathrm{~T}$ \\
\hline & & & & & & & $12-22-16$ & 18.13 & & $\mathrm{~T}$ \\
\hline & & & & & & & $02-24-17$ & 17.24 & & $\mathrm{~T}$ \\
\hline & & & & & & & $04-24-17$ & 18.41 & & $\mathrm{~T}$ \\
\hline & & & & & & & $07-3-17$ & 25.93 & & $\mathrm{~V}$ \\
\hline & & & & & & & $08-30-17$ & 37.98 & & $\mathrm{~V}$ \\
\hline & & & & & & & $10-31-17$ & 38.79 & & $\mathrm{~V}$ \\
\hline & & & & & & & $01-03-18$ & 17.26 & & $\mathrm{~V}$ \\
\hline & & & & & & & $02-22-18$ & 18.73 & & $\mathrm{~V}$ \\
\hline & & & & & & & $04-19-18$ & 17.70 & & $\mathrm{~V}$ \\
\hline \multirow{8}{*}{ 19N-04W-04E02 } & & & & & & & $07-03-17$ & 11.76 & & $\mathrm{~V}$ \\
\hline & & & & & & & $09-01-17$ & 18.50 & & $\mathrm{~V}$ \\
\hline & & & & & & & $10-31-17$ & 12.27 & & $\mathrm{~V}$ \\
\hline & & & & & & & $01-03-18$ & 10.37 & & $\mathrm{~V}$ \\
\hline & & & & & & & $02-22-18$ & 9.93 & & V \\
\hline & & & & & & & 04-19-18 & 13.16 & $\mathrm{R}$ & $\mathrm{V}$ \\
\hline & & & & & & & $06-28-18$ & 19.35 & & $\mathrm{~V}$ \\
\hline & & & & & & & $08-24-18$ & 19.21 & & $\mathrm{~V}$ \\
\hline \multirow[t]{13}{*}{ 19N-04W-02F02 } & 471002123090301 & 47.167 & -123.151 & 06-15-00 & 90 & 162 & $08-22-16$ & 19.01 & & $\mathrm{~T}$ \\
\hline & & & & & & & $10-27-16$ & 17.82 & & $\mathrm{~T}$ \\
\hline & & & & & & & $12-22-16$ & 6.50 & & $\mathrm{~T}$ \\
\hline & & & & & & & $02-24-17$ & 3.85 & & $\mathrm{~T}$ \\
\hline & & & & & & & $04-24-17$ & 3.73 & & $\mathrm{~T}$ \\
\hline & & & & & & & $07-03-17$ & 12.63 & & $\mathrm{~V}$ \\
\hline & & & & & & & $08-31-17$ & 17.56 & $\mathrm{R}$ & $\mathrm{V}$ \\
\hline & & & & & & & $10-31-17$ & 19.12 & & $\mathrm{~V}$ \\
\hline & & & & & & & 01-03-18 & 7.51 & & $\mathrm{~V}$ \\
\hline & & & & & & & $02-22-18$ & 5.22 & & $\mathrm{~V}$ \\
\hline & & & & & & & 04-19-18 & 7.33 & & $\mathrm{~V}$ \\
\hline & & & & & & & $06-28-18$ & 14.59 & & $\mathrm{~V}$ \\
\hline & & & & & & & $08-24-18$ & 18.76 & & $\mathrm{~V}$ \\
\hline
\end{tabular}


Table 2. Measured monthly groundwater levels, Mason County, western Washington, 2016-18.-Continued

\begin{tabular}{|c|c|c|c|c|c|c|c|c|c|c|}
\hline \multirow{2}{*}{ Local well No. } & \multirow{2}{*}{ Site No. } & \multirow{2}{*}{ Latitude } & \multirow{2}{*}{ Longitude } & \multirow{2}{*}{$\begin{array}{l}\text { Date of well } \\
\text { construction }\end{array}$} & \multirow{2}{*}{$\begin{array}{c}\text { Well } \\
\text { depth } \\
\text { (ft) }\end{array}$} & \multirow{2}{*}{$\begin{array}{l}\text { Land- } \\
\text { surface } \\
\text { altitude } \\
\text { (ft) }\end{array}$} & \multirow{2}{*}{$\begin{array}{l}\text { Measure- } \\
\text { ment } \\
\text { date }\end{array}$} & \multirow{2}{*}{$\begin{array}{c}\text { Groundwater } \\
\text { level } \\
\text { (feet below } \\
\text { land-surface) }\end{array}$} & \multicolumn{2}{|c|}{ Groundwater level } \\
\hline & & & & & & & & & Status & Method \\
\hline \multirow[t]{13}{*}{ 20N-03W-31J01 } & 471043123060301 & 47.179 & -123.101 & 05-05-97 & 119.6 & 135 & $08-25-16$ & 25.89 & & $\mathrm{~T}$ \\
\hline & & & & & & & $10-27-16$ & 25.04 & & $\mathrm{~T}$ \\
\hline & & & & & & & $12-27-16$ & 23.80 & & $\mathrm{~T}$ \\
\hline & & & & & & & $02-27-17$ & 21.84 & & $\mathrm{~T}$ \\
\hline & & & & & & & $04-21-17$ & 19.59 & & $\mathrm{~T}$ \\
\hline & & & & & & & $06-23-17$ & 22.30 & & V \\
\hline & & & & & & & $08-31-17$ & 25.08 & & $\mathrm{~V}$ \\
\hline & & & & & & & $10-27-17$ & 25.03 & & $\mathrm{~V}$ \\
\hline & & & & & & & 01-02-18 & 23.59 & & V \\
\hline & & & & & & & $02-23-18$ & 21.32 & & V \\
\hline & & & & & & & 04-20-18 & 21.29 & & $\mathrm{~V}$ \\
\hline & & & & & & & 06-26-18 & 23.89 & & V \\
\hline & & & & & & & 08-29-18 & 26.27 & & $\mathrm{~V}$ \\
\hline \multirow[t]{5}{*}{ 20N-04W-36A02 } & 471111123072601 & 47.186 & -123.124 & 01-19-99 & 246 & 281 & 08-24-16 & 164.31 & & $\mathrm{~T}$ \\
\hline & & & & & & & $10-27-16$ & 161.24 & & $\mathrm{~T}$ \\
\hline & & & & & & & $12-22-16$ & 160.03 & & $\mathrm{~T}$ \\
\hline & & & & & & & $02-24-17$ & 159.39 & & $\mathrm{~T}$ \\
\hline & & & & & & & $04-24-17$ & 158.90 & & $\mathrm{~T}$ \\
\hline \multirow[t]{11}{*}{$20 \mathrm{~N}-01 \mathrm{~W}-30 \mathrm{M} 02$} & 471129122514801 & 47.191 & -122.864 & $7-11-77$ & 309 & 262 & $09-22-16$ & 226.66 & & $\mathrm{~T}$ \\
\hline & & & & & & & $11-29-16$ & 226.40 & & $\mathrm{~T}$ \\
\hline & & & & & & & $01-26-17$ & 226.77 & & $\mathrm{~T}$ \\
\hline & & & & & & & 03-30-17 & 226.49 & & $\mathrm{~T}$ \\
\hline & & & & & & & $05-25-17$ & 226.28 & & $\mathrm{~V}$ \\
\hline & & & & & & & $07-21-17$ & 229.98 & $\mathrm{R}$ & $\mathrm{V}$ \\
\hline & & & & & & & $10-02-17$ & 226.09 & & $\mathrm{~V}$ \\
\hline & & & & & & & $11-20-17$ & 225.27 & & $\mathrm{~V}$ \\
\hline & & & & & & & 01-24-18 & 225.36 & & $\mathrm{~V}$ \\
\hline & & & & & & & 05-31-18 & 225.89 & & $\mathrm{~V}$ \\
\hline & & & & & & & $07-26-18$ & 227.12 & $\mathrm{R}$ & V \\
\hline \multirow[t]{13}{*}{$20 \mathrm{~N}-02 \mathrm{~W}-25 \mathrm{C} 01$} & 471148122523701 & 47.197 & -122.877 & 04-04-12 & 260 & 242 & 09-23-16 & 177.53 & & $\mathrm{~T}$ \\
\hline & & & & & & & $11-29-16$ & 175.93 & & $\mathrm{~T}$ \\
\hline & & & & & & & $01-26-17$ & 176.68 & & $\mathrm{~T}$ \\
\hline & & & & & & & 03-30-17 & 176.62 & & $\mathrm{~T}$ \\
\hline & & & & & & & $05-25-17$ & 175.29 & & $\mathrm{~V}$ \\
\hline & & & & & & & $07-21-17$ & 174.32 & & $\mathrm{~V}$ \\
\hline & & & & & & & $10-03-17$ & 173.67 & & $\mathrm{~V}$ \\
\hline & & & & & & & $11-20-17$ & 173.64 & & $\mathrm{~V}$ \\
\hline & & & & & & & 01-24-18 & 173.70 & & $\mathrm{~V}$ \\
\hline & & & & & & & 03-20-18 & 173.97 & & $\mathrm{~V}$ \\
\hline & & & & & & & 05-31-18 & 173.49 & & $\mathrm{~V}$ \\
\hline & & & & & & & $07-26-18$ & 173.49 & & V \\
\hline & & & & & & & $09-27-18$ & 173.45 & & $\mathrm{~V}$ \\
\hline
\end{tabular}


14 Groundwater and Surface-Water Data Collection for Mason County, Western Washington, 2016-18

Table 2. Measured monthly groundwater levels, Mason County, western Washington, 2016-18.-Continued

\begin{tabular}{|c|c|c|c|c|c|c|c|c|c|c|}
\hline \multirow{2}{*}{ Local well No. } & \multirow{2}{*}{ Site No. } & \multirow{2}{*}{ Latitude } & \multirow{2}{*}{ Longitude } & \multirow{2}{*}{$\begin{array}{l}\text { Date of well } \\
\text { construction }\end{array}$} & \multirow{2}{*}{$\begin{array}{c}\text { Well } \\
\text { depth } \\
\text { (ft) }\end{array}$} & \multirow{2}{*}{$\begin{array}{l}\text { Land- } \\
\text { surface } \\
\text { altitude } \\
\text { (ft) }\end{array}$} & \multirow{2}{*}{$\begin{array}{c}\text { Measure- } \\
\text { ment } \\
\text { date }\end{array}$} & \multirow{2}{*}{$\begin{array}{c}\text { Groundwater } \\
\text { level } \\
\text { (feet below } \\
\text { land-surface) }\end{array}$} & \multicolumn{2}{|c|}{ Groundwater level } \\
\hline & & & & & & & & & Status & Method \\
\hline \multirow[t]{13}{*}{$20 \mathrm{~N}-04 \mathrm{~W}-25 \mathrm{E} 01$} & 471148123081801 & 47.197 & -123.138 & 03-18-99 & 65 & 86 & $08-24-16$ & 8.12 & & $\mathrm{~T}$ \\
\hline & & & & & & & $10-27-16$ & 4.16 & & $\mathrm{~T}$ \\
\hline & & & & & & & $12-27-16$ & 2.57 & & $\mathrm{~T}$ \\
\hline & & & & & & & $02-27-17$ & 1.58 & & $\mathrm{~T}$ \\
\hline & & & & & & & $04-21-17$ & 0.80 & & $\mathrm{~T}$ \\
\hline & & & & & & & $06-23-17$ & 4.09 & & $\mathrm{~V}$ \\
\hline & & & & & & & $08-31-17$ & 7.09 & & $\mathrm{~V}$ \\
\hline & & & & & & & $10-27-17$ & 6.02 & & V \\
\hline & & & & & & & $01-02-18$ & 2.05 & & V \\
\hline & & & & & & & $02-23-18$ & 2.00 & & $\mathrm{~V}$ \\
\hline & & & & & & & $04-20-18$ & 1.80 & & $\mathrm{~V}$ \\
\hline & & & & & & & $06-26-18$ & 5.99 & & $\mathrm{~V}$ \\
\hline & & & & & & & $08-29-18$ & 8.35 & & V \\
\hline \multirow[t]{13}{*}{ 20N-06W-29A01 } & 471148123272001 & 47.197 & -123.456 & 08-16-07 & 35 & 349 & $08-23-16$ & 11.93 & & $\mathrm{~T}$ \\
\hline & & & & & & & $10-24-16$ & 2.61 & & $\mathrm{~T}$ \\
\hline & & & & & & & $12-21-16$ & 0.03 & & $\mathrm{~T}$ \\
\hline & & & & & & & $02-28-17$ & 0.34 & & $\mathrm{~T}$ \\
\hline & & & & & & & $05-05-17$ & 0.41 & & $\mathrm{~T}$ \\
\hline & & & & & & & $06-26-17$ & 1.59 & & $\mathrm{~V}$ \\
\hline & & & & & & & 09-01-17 & 7.32 & & $\mathrm{~V}$ \\
\hline & & & & & & & $10-30-17$ & 9.26 & & V \\
\hline & & & & & & & 01-05-18 & -0.01 & & $\mathrm{~V}$ \\
\hline & & & & & & & $02-20-18$ & 0.31 & & $\mathrm{~V}$ \\
\hline & & & & & & & 04-24-18 & 0.44 & & $\mathrm{~V}$ \\
\hline & & & & & & & $06-27-18$ & 3.11 & & V \\
\hline & & & & & & & $08-30-18$ & 11.00 & & $\mathrm{~V}$ \\
\hline \multirow[t]{13}{*}{$20 \mathrm{~N}-04 \mathrm{~W}-27 \mathrm{~A} 03$} & 471154123095901 & 47.198 & -123.166 & 09-02-98 & 100 & 366 & $08-24-16$ & 70.26 & & $\mathrm{~T}$ \\
\hline & & & & & & & $10-28-16$ & 72.71 & & $\mathrm{~T}$ \\
\hline & & & & & & & $12-27-16$ & 72.14 & & $\mathrm{~T}$ \\
\hline & & & & & & & $02-28-17$ & 70.87 & $\mathrm{R}$ & $\mathrm{T}$ \\
\hline & & & & & & & $04-21-17$ & 65.79 & & $\mathrm{~T}$ \\
\hline & & & & & & & $06-20-17$ & 65.71 & & V \\
\hline & & & & & & & 08-31-17 & 68.69 & & V \\
\hline & & & & & & & $10-27-17$ & 71.08 & & $\mathrm{~V}$ \\
\hline & & & & & & & 01-02-18 & 72.03 & & $\mathrm{~V}$ \\
\hline & & & & & & & $02-23-18$ & 70.10 & & V \\
\hline & & & & & & & $04-20-18$ & 69.16 & & V \\
\hline & & & & & & & $06-26-18$ & 69.34 & & V \\
\hline & & & & & & & 08-29-18 & 77.18 & $\mathrm{P}$ & $\mathrm{V}$ \\
\hline \multirow[t]{14}{*}{$20 \mathrm{~N}-05 \mathrm{~W}-25 \mathrm{~A} 01$} & 471155123145201 & 47.199 & -123.248 & 06-19-93 & 80 & 250 & 08-05-16 & 7.00 & & $\mathrm{~V}$ \\
\hline & & & & & & & $08-23-16$ & 7.18 & & $\mathrm{~T}$ \\
\hline & & & & & & & $10-25-16$ & 3.46 & & $\mathrm{~T}$ \\
\hline & & & & & & & $12-27-16$ & 2.54 & & $\mathrm{~T}$ \\
\hline & & & & & & & $02-27-17$ & 2.79 & & $\mathrm{~T}$ \\
\hline & & & & & & & $04-21-17$ & 2.70 & & $\mathrm{~T}$ \\
\hline & & & & & & & $06-20-17$ & 5.06 & & $\mathrm{~V}$ \\
\hline & & & & & & & $08-31-17$ & 6.91 & & V \\
\hline & & & & & & & $10-27-17$ & 5.68 & & V \\
\hline & & & & & & & $01-02-18$ & 2.38 & & V \\
\hline & & & & & & & $02-23-18$ & 3.22 & & $\mathrm{~V}$ \\
\hline & & & & & & & $04-20-18$ & 2.67 & & $\mathrm{~V}$ \\
\hline & & & & & & & $06-21-18$ & 6.24 & & $\mathrm{~V}$ \\
\hline & & & & & & & $08-29-18$ & 7.21 & & V \\
\hline
\end{tabular}


Table 2. Measured monthly groundwater levels, Mason County, western Washington, 2016-18.-Continued

\begin{tabular}{|c|c|c|c|c|c|c|c|c|c|c|}
\hline \multirow{2}{*}{ Local well No. } & \multirow{2}{*}{ Site No. } & \multirow{2}{*}{ Latitude } & \multirow{2}{*}{ Longitude } & \multirow{2}{*}{$\begin{array}{l}\text { Date of well } \\
\text { construction }\end{array}$} & \multirow{2}{*}{$\begin{array}{c}\text { Well } \\
\text { depth } \\
\text { (ft) }\end{array}$} & \multirow{2}{*}{$\begin{array}{l}\text { Land- } \\
\text { surface } \\
\text { altitude } \\
\text { (ft) }\end{array}$} & \multirow{2}{*}{$\begin{array}{c}\text { Measure- } \\
\text { ment } \\
\text { date }\end{array}$} & \multirow{2}{*}{$\begin{array}{l}\text { Groundwater } \\
\text { level } \\
\text { (feet below } \\
\text { land-surface) }\end{array}$} & \multicolumn{2}{|c|}{ Groundwater level } \\
\hline & & & & & & & & & Status & Method \\
\hline $20 \mathrm{~N}-03 \mathrm{~W}-21 \mathrm{M} 03$ & 471219123042701 & 47.205 & -123.074 & 07-10-92 & 59 & 21 & $\begin{array}{l}08-25-16 \\
10-27-16 \\
12-27-16 \\
02-27-17 \\
04-21-17 \\
06-23-17 \\
08-31-17 \\
10-27-17 \\
01-04-18 \\
02-23-18 \\
04-20-18 \\
06-26-18 \\
08-29-18\end{array}$ & $\begin{array}{l}.83 \\
.89 \\
.89 \\
.88 \\
.92 \\
.88 \\
.90 \\
.86 \\
.92 \\
.71 \\
.91 \\
.91 \\
.92\end{array}$ & & $\begin{array}{l}\mathrm{T} \\
\mathrm{T} \\
\mathrm{T} \\
\mathrm{T} \\
\mathrm{S} \\
\mathrm{S} \\
\mathrm{S} \\
\mathrm{S} \\
\mathrm{S} \\
\mathrm{S} \\
\mathrm{S} \\
\mathrm{S} \\
\mathrm{S}\end{array}$ \\
\hline $20 \mathrm{~N}-03 \mathrm{~W}-23 \mathrm{~F} 03$ & 471233123013401 & 47.209 & -123.026 & $12-12-96$ & 53.6 & 42 & $\begin{array}{l}09-23-16 \\
12-01-16 \\
01-26-17 \\
03-30-17 \\
05-25-17 \\
07-20-17 \\
10-03-17 \\
11-20-17 \\
01-24-18 \\
03-20-18 \\
05-31-18 \\
07-26-18 \\
09-27-18\end{array}$ & $\begin{array}{l}28.36 \\
27.68 \\
26.66 \\
24.52 \\
25.54 \\
27.13 \\
28.08 \\
28.06 \\
25.45 \\
26.12 \\
26.72 \\
28.07 \\
28.39\end{array}$ & & $\begin{array}{l}\mathrm{T} \\
\mathrm{T} \\
\mathrm{T} \\
\mathrm{T} \\
\mathrm{V} \\
\mathrm{V} \\
\mathrm{V} \\
\mathrm{V} \\
\mathrm{V} \\
\mathrm{V} \\
\mathrm{V} \\
\mathrm{V} \\
\mathrm{V}\end{array}$ \\
\hline $20 \mathrm{~N}-06 \mathrm{~W}-17 \mathrm{~N} 01$ & 471256123282101 & 47.216 & -123.472 & $05-15-07$ & 63 & 367 & $\begin{array}{l}08-23-16 \\
10-24-16 \\
12-21-16 \\
02-28-17 \\
05-05-17 \\
06-26-17 \\
09-01-17 \\
10-30-17 \\
01-05-18 \\
02-20-18 \\
04-24-18 \\
06-27-18 \\
08-30-18\end{array}$ & $\begin{array}{l}6.74 \\
2.37 \\
1.39 \\
1.89 \\
1.87 \\
2.60 \\
4.87 \\
5.12 \\
1.42 \\
1.75 \\
2.04 \\
3.49 \\
6.54\end{array}$ & $\mathrm{R}$ & $\begin{array}{l}\mathrm{T} \\
\mathrm{T} \\
\mathrm{T} \\
\mathrm{T} \\
\mathrm{T} \\
\mathrm{V} \\
\mathrm{V} \\
\mathrm{V} \\
\mathrm{V} \\
\mathrm{V} \\
\mathrm{V} \\
\mathrm{V} \\
\mathrm{V}\end{array}$ \\
\hline $20 \mathrm{~N}-02 \mathrm{~W}-14 \mathrm{~F} 01$ & 471330122540601 & 47.225 & -122.902 & $05-17-13$ & 85 & 152 & $\begin{array}{l}11-29-16 \\
01-26-17 \\
03-30-17 \\
05-26-17\end{array}$ & $\begin{array}{l}59.42 \\
28.49 \\
25.87 \\
27.70\end{array}$ & $\begin{array}{l}\mathrm{P} \\
\mathrm{R} \\
\mathrm{P}\end{array}$ & $\begin{array}{l}\mathrm{T} \\
\mathrm{T} \\
\mathrm{T} \\
\mathrm{T}\end{array}$ \\
\hline
\end{tabular}


Table 2. Measured monthly groundwater levels, Mason County, western Washington, 2016-18._Continued

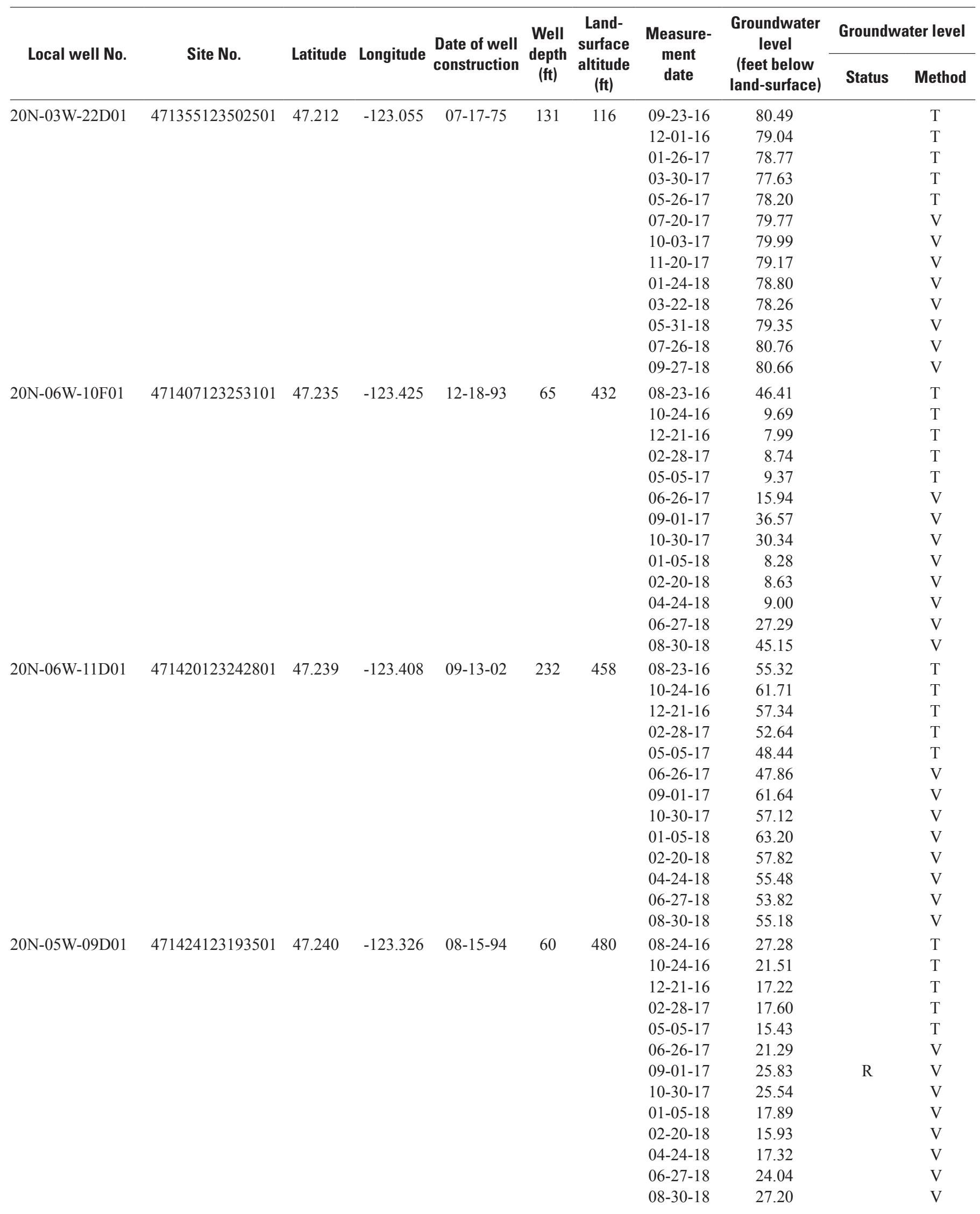


Table 2. Measured monthly groundwater levels, Mason County, western Washington, 2016-18.-Continued

\begin{tabular}{|c|c|c|c|c|c|c|c|c|c|c|}
\hline \multirow{2}{*}{ Local well No. } & \multirow{2}{*}{ Site No. } & \multirow{2}{*}{ Latitude } & \multirow{2}{*}{ Longitude } & \multirow{2}{*}{$\begin{array}{l}\text { Date of well } \\
\text { construction }\end{array}$} & \multirow{2}{*}{$\begin{array}{l}\text { Well } \\
\text { depth } \\
\text { (ft) }\end{array}$} & \multirow{2}{*}{$\begin{array}{l}\text { Land- } \\
\text { surface } \\
\text { altitude } \\
\text { (ft) }\end{array}$} & \multirow{2}{*}{$\begin{array}{l}\text { Measure- } \\
\text { ment } \\
\text { date }\end{array}$} & \multirow{2}{*}{$\begin{array}{c}\text { Groundwater } \\
\text { level } \\
\text { (feet below } \\
\text { land-surface) }\end{array}$} & \multicolumn{2}{|c|}{ Groundwater level } \\
\hline & & & & & & & & & Status & Method \\
\hline \multirow{13}{*}{$20 \mathrm{~N}-03 \mathrm{~W}-02 \mathrm{P} 01$} & 471439123012901 & 47.244 & -123.025 & $12-28-92$ & 59 & 162 & $09-21-16$ & 31.08 & & $\mathrm{~T}$ \\
\hline & & & & & & & $11-30-16$ & 21.58 & & $\mathrm{~T}$ \\
\hline & & & & & & & $01-24-17$ & 21.38 & & $\mathrm{~T}$ \\
\hline & & & & & & & $03-27-17$ & 20.72 & & $\mathrm{~T}$ \\
\hline & & & & & & & $05-24-17$ & 21.89 & & $\mathrm{~V}$ \\
\hline & & & & & & & $07-20-17$ & 26.01 & $\mathrm{R}$ & V \\
\hline & & & & & & & $09-28-17$ & 28.46 & & $\mathrm{~V}$ \\
\hline & & & & & & & $11-30-17$ & 21.71 & & $\mathrm{~V}$ \\
\hline & & & & & & & $01-25-18$ & 22.99 & $\mathrm{P}$ & $\mathrm{V}$ \\
\hline & & & & & & & 03-19-18 & 21.82 & & $\mathrm{~V}$ \\
\hline & & & & & & & $05-29-18$ & 23.01 & & V \\
\hline & & & & & & & $07-23-18$ & 26.31 & & V \\
\hline & & & & & & & $09-24-18$ & 30.17 & $\mathrm{R}$ & $\mathrm{V}$ \\
\hline \multirow{13}{*}{$20 \mathrm{~N}-03 \mathrm{~W}-04 \mathrm{P} 02$} & 471443123035601 & 47.245 & -123.066 & $11-16-05$ & 70 & 201 & $08-25-16$ & 21.95 & & $\mathrm{~T}$ \\
\hline & & & & & & & $10-27-16$ & 17.49 & & $\mathrm{~T}$ \\
\hline & & & & & & & $12-23-16$ & 17.66 & & $\mathrm{~T}$ \\
\hline & & & & & & & $02-27-17$ & 17.91 & & $\mathrm{~T}$ \\
\hline & & & & & & & $04-21-17$ & 17.74 & & $\mathrm{~T}$ \\
\hline & & & & & & & $06-20-17$ & 19.25 & & $\mathrm{~V}$ \\
\hline & & & & & & & $08-31-17$ & 21.21 & & $\mathrm{~V}$ \\
\hline & & & & & & & $10-27-17$ & 22.27 & $\mathrm{R}$ & $\mathrm{V}$ \\
\hline & & & & & & & $01-02-18$ & 17.49 & & $\mathrm{~V}$ \\
\hline & & & & & & & $02-23-18$ & 18.29 & & V \\
\hline & & & & & & & 04-20-18 & 17.69 & & $\mathrm{~V}$ \\
\hline & & & & & & & $06-29-18$ & 20.47 & & $\mathrm{~V}$ \\
\hline & & & & & & & $08-29-18$ & 23.51 & $\mathrm{R}$ & V \\
\hline \multirow{13}{*}{$\begin{array}{l}\text { 20N-03W- } \\
04 \mathrm{~W} 04 \mathrm{D} 1\end{array}$} & 471445123034201 & 47.246 & -123.062 & $02-01-95$ & 341 & 200 & $08-25-16$ & 114.01 & & $\mathrm{~T}$ \\
\hline & & & & & & & $10-27-16$ & 113.31 & & $\mathrm{~T}$ \\
\hline & & & & & & & $12-27-16$ & 112.31 & & $\mathrm{~T}$ \\
\hline & & & & & & & $02-27-17$ & 111.88 & & $\mathrm{~T}$ \\
\hline & & & & & & & $04-21-17$ & 110.91 & & $\mathrm{~T}$ \\
\hline & & & & & & & $06-20-17$ & 111.18 & & $\mathrm{~V}$ \\
\hline & & & & & & & $08-31-17$ & 113.14 & & $\mathrm{~V}$ \\
\hline & & & & & & & $10-27-17$ & 113.05 & & V \\
\hline & & & & & & & 01-04-18 & 112.14 & & $\mathrm{~V}$ \\
\hline & & & & & & & $02-23-18$ & 111.70 & & $\mathrm{~V}$ \\
\hline & & & & & & & $04-20-18$ & 111.56 & & $\mathrm{~V}$ \\
\hline & & & & & & & $06-21-18$ & 112.74 & & $\mathrm{~V}$ \\
\hline & & & & & & & 08-29-18 & 114.06 & & $\mathrm{~V}$ \\
\hline \multirow[t]{13}{*}{ 20N-04W-04K01 } & 471504123113701 & 47.251 & -123.194 & $03-30-98$ & 115 & 319 & $08-26-16$ & 38.84 & & $\mathrm{~T}$ \\
\hline & & & & & & & $10-25-16$ & 34.51 & & $\mathrm{~T}$ \\
\hline & & & & & & & $12-22-16$ & 27.44 & & $\mathrm{~T}$ \\
\hline & & & & & & & $02-24-17$ & 24.24 & & $\mathrm{~T}$ \\
\hline & & & & & & & $04-24-17$ & 22.47 & & $\mathrm{~T}$ \\
\hline & & & & & & & $06-30-17$ & 28.91 & & $\mathrm{~V}$ \\
\hline & & & & & & & $08-30-17$ & 36.41 & & $\mathrm{~V}$ \\
\hline & & & & & & & $10-31-17$ & 40.82 & & $\mathrm{~V}$ \\
\hline & & & & & & & 01-03-18 & 28.10 & & $\mathrm{~V}$ \\
\hline & & & & & & & $02-22-18$ & 25.17 & & $\mathrm{~V}$ \\
\hline & & & & & & & 04-19-18 & 25.30 & & $\mathrm{~V}$ \\
\hline & & & & & & & $06-28-18$ & 34.25 & & $\mathrm{~V}$ \\
\hline & & & & & & & $08-24-18$ & 39.43 & & $\mathrm{~V}$ \\
\hline
\end{tabular}


Table 2. Measured monthly groundwater levels, Mason County, western Washington, 2016-18.-Continued

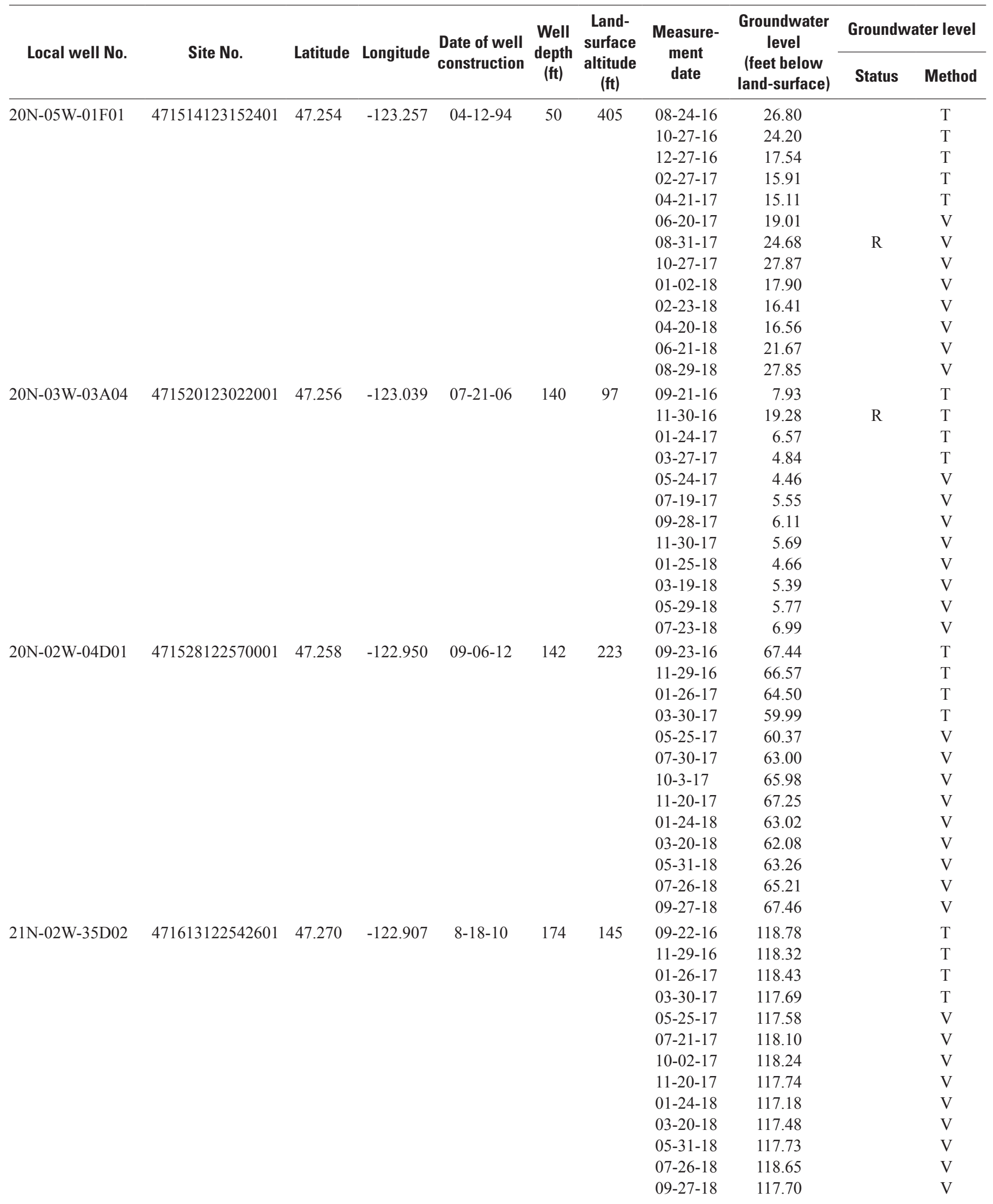


Table 2. Measured monthly groundwater levels, Mason County, western Washington, 2016-18.-Continued

\begin{tabular}{|c|c|c|c|c|c|c|c|c|c|c|}
\hline \multirow{2}{*}{ Local well No. } & \multirow{2}{*}{ Site No. } & \multirow{2}{*}{ Latitude } & \multirow{2}{*}{ Longitude } & \multirow{2}{*}{$\begin{array}{l}\text { Date of well } \\
\text { construction }\end{array}$} & \multirow{2}{*}{$\begin{array}{c}\text { Well } \\
\text { depth } \\
\text { (ft) }\end{array}$} & \multirow{2}{*}{$\begin{array}{l}\text { Land- } \\
\text { surface } \\
\text { altitude } \\
\text { (ft) }\end{array}$} & \multirow{2}{*}{$\begin{array}{c}\text { Measure- } \\
\text { ment } \\
\text { date }\end{array}$} & \multirow{2}{*}{$\begin{array}{l}\text { Groundwater } \\
\text { level } \\
\text { (feet below } \\
\text { land-surface) }\end{array}$} & \multicolumn{2}{|c|}{ Groundwater level } \\
\hline & & & & & & & & & Status & Method \\
\hline \multirow[t]{13}{*}{$21 \mathrm{~N}-03 \mathrm{~W}-36 \mathrm{~A} 01$} & 471620122595201 & 47.272 & -122.998 & $6-13-05$ & 160 & 142 & $09-21-16$ & 111.25 & & $\mathrm{~T}$ \\
\hline & & & & & & & $12-01-16$ & 109.93 & & $\mathrm{~T}$ \\
\hline & & & & & & & $01-24-17$ & 109.75 & & $\mathrm{~T}$ \\
\hline & & & & & & & $03-27-17$ & 108.74 & & $\mathrm{~T}$ \\
\hline & & & & & & & $05-24-17$ & 109.33 & & $\mathrm{~V}$ \\
\hline & & & & & & & $07-20-17$ & 111.07 & & V \\
\hline & & & & & & & $09-28-17$ & 110.97 & & $\mathrm{~V}$ \\
\hline & & & & & & & $11-30-17$ & 109.68 & & V \\
\hline & & & & & & & $01-25-18$ & 109.06 & & $\mathrm{~V}$ \\
\hline & & & & & & & 03-19-18 & 109.53 & & $\mathrm{~V}$ \\
\hline & & & & & & & $05-29-18$ & 110.63 & & $\mathrm{~V}$ \\
\hline & & & & & & & $07-23-18$ & 112.84 & & $\mathrm{~V}$ \\
\hline & & & & & & & 09-24-18 & 111.58 & & V \\
\hline \multirow{13}{*}{$21 \mathrm{~N}-03 \mathrm{~W}-30 \mathrm{~J} 04$} & 471636123060901 & 47.277 & -123.103 & $8-16-06$ & 188 & 324 & $09-20-16$ & 104.78 & & $\mathrm{~T}$ \\
\hline & & & & & & & 12-1-16 & 103.33 & & $\mathrm{~T}$ \\
\hline & & & & & & & $01-26-17$ & 99.78 & & $\mathrm{~T}$ \\
\hline & & & & & & & $03-27-17$ & 96.83 & & $\mathrm{~T}$ \\
\hline & & & & & & & $05-24-17$ & 98.21 & & $\mathrm{~V}$ \\
\hline & & & & & & & 07-19-17 & 101.53 & & $\mathrm{~V}$ \\
\hline & & & & & & & $09-27-17$ & 103.09 & & V \\
\hline & & & & & & & $11-30-17$ & 103.24 & & $\mathrm{~V}$ \\
\hline & & & & & & & $01-25-18$ & 100.35 & & $\mathrm{~V}$ \\
\hline & & & & & & & 03-19-18 & 99.98 & & $\mathrm{~V}$ \\
\hline & & & & & & & $05-29-18$ & 101.58 & & V \\
\hline & & & & & & & $07-23-18$ & 103.19 & & V \\
\hline & & & & & & & 09-24-18 & 104.71 & & V \\
\hline \multirow[t]{13}{*}{$21 \mathrm{~N}-02 \mathrm{~W}-25 \mathrm{~F} 02$} & 471659122523901 & 47.283 & -122.877 & $07-25-12$ & 151 & 47 & $09-22-16$ & 59.39 & & $\mathrm{~T}$ \\
\hline & & & & & & & $11-29-16$ & 58.52 & & $\mathrm{~T}$ \\
\hline & & & & & & & $01-26-17$ & 58.37 & & $\mathrm{~T}$ \\
\hline & & & & & & & $03-30-17$ & 57.49 & & $\mathrm{~T}$ \\
\hline & & & & & & & $05-25-17$ & 58.15 & & V \\
\hline & & & & & & & $07-21-17$ & 59.37 & & $\mathrm{~V}$ \\
\hline & & & & & & & $10-02-17$ & 59.11 & & $\mathrm{~V}$ \\
\hline & & & & & & & $11-20-17$ & 58.45 & & V \\
\hline & & & & & & & $01-24-18$ & 57.34 & & $\mathrm{~V}$ \\
\hline & & & & & & & $03-20-18$ & 58.01 & & $\mathrm{~V}$ \\
\hline & & & & & & & 05-31-18 & 58.68 & & $\mathrm{~V}$ \\
\hline & & & & & & & $07-26-18$ & 59.72 & & $\mathrm{~V}$ \\
\hline & & & & & & & $09-27-18$ & 59.08 & & V \\
\hline \multirow[t]{13}{*}{$21 \mathrm{~N}-06 \mathrm{~W}-27 \mathrm{H} 01$} & 471701123252801 & 47.284 & -123.424 & $11-5-92$ & 67.75 & 625 & $08-29-16$ & 57.42 & & $\mathrm{~T}$ \\
\hline & & & & & & & $10-24-16$ & 46.74 & & $\mathrm{~T}$ \\
\hline & & & & & & & $12-21-16$ & 46.67 & & $\mathrm{~T}$ \\
\hline & & & & & & & $02-28-17$ & 48.17 & & $\mathrm{~T}$ \\
\hline & & & & & & & 05-05-17 & 48.39 & & $\mathrm{~T}$ \\
\hline & & & & & & & $06-26-17$ & 49.29 & & $\mathrm{~V}$ \\
\hline & & & & & & & 09-01-17 & 54.47 & & $\mathrm{~V}$ \\
\hline & & & & & & & $10-30-17$ & 48.57 & & V \\
\hline & & & & & & & 01-05-18 & 47.56 & & V \\
\hline & & & & & & & $02-20-18$ & 48.18 & & $\mathrm{~V}$ \\
\hline & & & & & & & 04-24-18 & 47.92 & & $\mathrm{~V}$ \\
\hline & & & & & & & $06-27-18$ & 51.18 & & $\mathrm{~V}$ \\
\hline & & & & & & & $08-30-18$ & 59.46 & & V \\
\hline
\end{tabular}


Table 2. Measured monthly groundwater levels, Mason County, western Washington, 2016-18._Continued

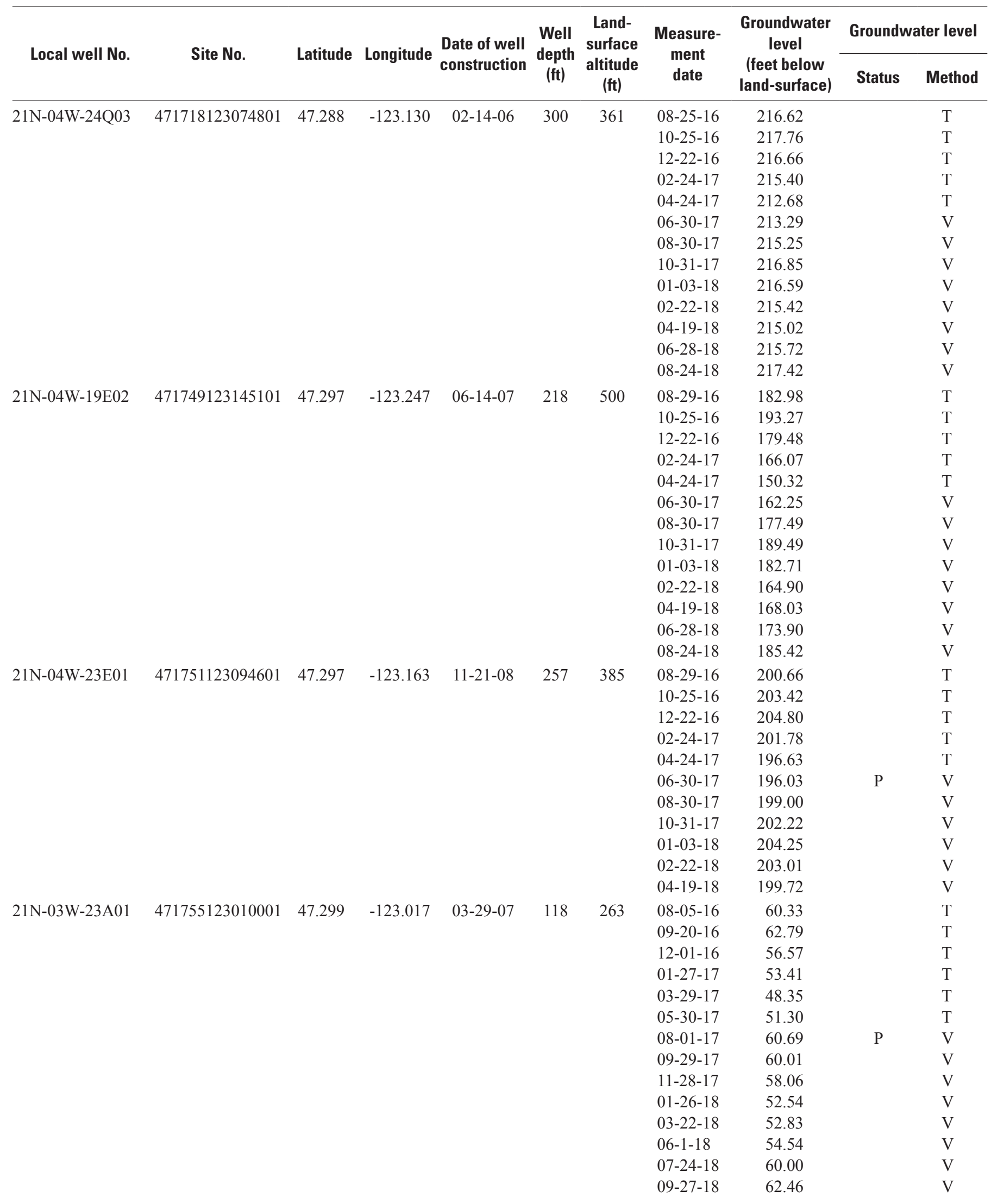


Table 2. Measured monthly groundwater levels, Mason County, western Washington, 2016-18.-Continued

\begin{tabular}{|c|c|c|c|c|c|c|c|c|c|c|}
\hline \multirow{2}{*}{ Local well No. } & \multirow{2}{*}{ Site No. } & \multirow{2}{*}{ Latitude } & \multirow{2}{*}{ Longitude } & \multirow{2}{*}{$\begin{array}{l}\text { Date of well } \\
\text { construction }\end{array}$} & \multirow{2}{*}{$\begin{array}{l}\text { Well } \\
\text { depth } \\
\text { (ft) }\end{array}$} & \multirow{2}{*}{$\begin{array}{l}\text { Land- } \\
\text { surface } \\
\text { altitude } \\
\text { (ft) }\end{array}$} & \multirow{2}{*}{$\begin{array}{c}\text { Measure- } \\
\text { ment } \\
\text { date }\end{array}$} & \multirow{2}{*}{$\begin{array}{c}\text { Groundwater } \\
\text { level } \\
\text { (feet below } \\
\text { land-surface) }\end{array}$} & \multicolumn{2}{|c|}{ Groundwater level } \\
\hline & & & & & & & & & Status & Method \\
\hline \multirow[t]{13}{*}{$21 \mathrm{~N}-02 \mathrm{~W}-14 \mathrm{R} 01$} & 471810122533101 & 47.303 & -122.892 & $10-22-10$ & 193 & 186 & $09-22-16$ & 151.96 & & $\mathrm{~T}$ \\
\hline & & & & & & & $11-28-16$ & 151.78 & & $\mathrm{~T}$ \\
\hline & & & & & & & $01-27-17$ & 151.29 & & $\mathrm{~T}$ \\
\hline & & & & & & & $03-28-17$ & 150.97 & & $\mathrm{~T}$ \\
\hline & & & & & & & $05-26-17$ & 150.50 & & $\mathrm{~T}$ \\
\hline & & & & & & & $07-28-17$ & 151.17 & & V \\
\hline & & & & & & & 09-28-17 & 151.32 & & V \\
\hline & & & & & & & $11-22-17$ & 151.05 & & $\mathrm{~V}$ \\
\hline & & & & & & & 01-29-18 & 150.73 & & V \\
\hline & & & & & & & $03-20-18$ & 150.83 & & V \\
\hline & & & & & & & $05-30-18$ & 151.02 & & V \\
\hline & & & & & & & $07-25-18$ & 151.64 & & V \\
\hline & & & & & & & 09-25-18 & 151.83 & & $\mathrm{~V}$ \\
\hline \multirow[t]{13}{*}{$21 \mathrm{~N}-05 \mathrm{~W}-11 \mathrm{~A} 01$} & 471939123162601 & 47.328 & -123.274 & 04-04-07 & 44 & 119 & 09-20-16 & 9.06 & & $\mathrm{~T}$ \\
\hline & & & & & & & $11-30-16$ & 6.24 & & $\mathrm{~T}$ \\
\hline & & & & & & & $01-24-17$ & 6.83 & & $\mathrm{~T}$ \\
\hline & & & & & & & $03-27-17$ & 6.29 & & $\mathrm{~T}$ \\
\hline & & & & & & & $05-24-17$ & 7.74 & & V \\
\hline & & & & & & & 07-19-17 & 8.84 & $\mathrm{R}$ & V \\
\hline & & & & & & & $10-02-17$ & 9.34 & & $\mathrm{~V}$ \\
\hline & & & & & & & $11-30-17$ & 6.27 & & V \\
\hline & & & & & & & $01-25-18$ & 4.93 & & V \\
\hline & & & & & & & 03-19-18 & 8.08 & & $\mathrm{~V}$ \\
\hline & & & & & & & $05-29-18$ & 9.37 & & $\mathrm{~V}$ \\
\hline & & & & & & & $07-23-18$ & 9.14 & & V \\
\hline & & & & & & & 09-24-18 & 9.24 & & $\mathrm{~V}$ \\
\hline \multirow[t]{13}{*}{$21 \mathrm{~N}-04 \mathrm{~W}-07 \mathrm{C} 01$} & 471946123142901 & 47.330 & -123.241 & $07-10-14$ & 80 & 79 & 08-29-16 & 14.10 & & $\mathrm{~T}$ \\
\hline & & & & & & & $10-25-16$ & 9.14 & & $\mathrm{~T}$ \\
\hline & & & & & & & $12-22-16$ & 12.18 & & $\mathrm{~T}$ \\
\hline & & & & & & & $02-24-17$ & 10.50 & & $\mathrm{~T}$ \\
\hline & & & & & & & 04-24-17 & 12.07 & & $\mathrm{~T}$ \\
\hline & & & & & & & $06-30-17$ & 13.51 & & V \\
\hline & & & & & & & 08-30-17 & 14.07 & & V \\
\hline & & & & & & & $10-31-17$ & 13.38 & & V \\
\hline & & & & & & & 01-03-18 & 12.29 & & $\mathrm{~V}$ \\
\hline & & & & & & & $02-22-18$ & 12.88 & & $\mathrm{~V}$ \\
\hline & & & & & & & 04-19-18 & 12.34 & & $\mathrm{~V}$ \\
\hline & & & & & & & $06-28-18$ & 13.72 & & $\mathrm{~V}$ \\
\hline & & & & & & & 08-24-18 & 14.19 & & $\mathrm{~V}$ \\
\hline \multirow[t]{13}{*}{$21 \mathrm{~N}-02 \mathrm{~W}-03 \mathrm{E} 01$} & 472024122554001 & 47.340 & -122.928 & 05-21-09 & 167 & 239 & 09-23-16 & 115.55 & & $\mathrm{~T}$ \\
\hline & & & & & & & $11-28-16$ & 115.05 & & $\mathrm{~T}$ \\
\hline & & & & & & & $01-27-17$ & 114.88 & & $\mathrm{~T}$ \\
\hline & & & & & & & $03-28-17$ & 141.78 & & $\mathrm{~T}$ \\
\hline & & & & & & & $05-26-17$ & 112.10 & & $\mathrm{~T}$ \\
\hline & & & & & & & $07-28-17$ & 112.85 & & V \\
\hline & & & & & & & 09-28-17 & 113.50 & & V \\
\hline & & & & & & & $11-27-17$ & 113.87 & & $\mathrm{~V}$ \\
\hline & & & & & & & $01-25-18$ & 112.92 & & $\mathrm{~V}$ \\
\hline & & & & & & & 03-20-18 & 112.86 & & $\mathrm{~V}$ \\
\hline & & & & & & & $05-30-18$ & 113.05 & & V \\
\hline & & & & & & & $07-25-18$ & 114.08 & & V \\
\hline & & & & & & & $09-25-18$ & 114.93 & & $\mathrm{~V}$ \\
\hline
\end{tabular}




\section{Groundwater and Surface-Water Data Collection for Mason County, Western Washington, 2016-18}

Table 2. Measured monthly groundwater levels, Mason County, western Washington, 2016-18.-Continued

\begin{tabular}{|c|c|c|c|c|c|c|c|c|c|c|}
\hline \multirow{2}{*}{ Local well No. } & \multirow{2}{*}{ Site No. } & \multirow{2}{*}{ Latitude } & \multirow{2}{*}{ Longitude } & \multirow{2}{*}{$\begin{array}{l}\text { Date of well } \\
\text { construction }\end{array}$} & \multirow{2}{*}{$\begin{array}{c}\text { Well } \\
\text { depth } \\
\text { (ft) }\end{array}$} & \multirow{2}{*}{$\begin{array}{l}\text { Land- } \\
\text { surface } \\
\text { altitude } \\
\text { (ft) }\end{array}$} & \multirow{2}{*}{$\begin{array}{l}\text { Measure- } \\
\text { ment } \\
\text { date }\end{array}$} & \multirow{2}{*}{$\begin{array}{c}\text { Groundwater } \\
\text { level } \\
\text { (feet below } \\
\text { land-surface) }\end{array}$} & \multicolumn{2}{|c|}{ Groundwater level } \\
\hline & & & & & & & & & Status & Method \\
\hline \multirow[t]{8}{*}{$22 \mathrm{~N}-03 \mathrm{~W}-31 \mathrm{Q} 02$} & \multirow[t]{8}{*}{472052123063001} & \multirow[t]{8}{*}{47.348} & \multirow[t]{8}{*}{-123.108} & \multirow[t]{8}{*}{ 11-10-08 } & \multirow[t]{8}{*}{133} & \multirow[t]{8}{*}{13} & $09-20-16$ & 4.34 & & $\mathrm{~T}$ \\
\hline & & & & & & & $11-29-16$ & 3.91 & & $\mathrm{~T}$ \\
\hline & & & & & & & $01-27-17$ & 4.59 & & $\mathrm{~T}$ \\
\hline & & & & & & & $07-31-17$ & 5.83 & & $\mathrm{~V}$ \\
\hline & & & & & & & 09-29-17 & 4.79 & & V \\
\hline & & & & & & & $11-28-17$ & 3.94 & & $\mathrm{~V}$ \\
\hline & & & & & & & $01-25-18$ & 4.35 & & $\mathrm{~V}$ \\
\hline & & & & & & & $03-23-18$ & 5.71 & & $\mathrm{~V}$ \\
\hline \multirow{11}{*}{$22 \mathrm{~N}-02 \mathrm{~W}-33 \mathrm{M} 02$} & & & & & & & $11-28-16$ & 73.68 & & $\mathrm{~T}$ \\
\hline & & & & & & & $01-27-17$ & 72.79 & & $\mathrm{~T}$ \\
\hline & & & & & & & $03-28-17$ & 68.95 & & $\mathrm{~T}$ \\
\hline & & & & & & & $05-26-17$ & 66.10 & & $\mathrm{~T}$ \\
\hline & & & & & & & $07-28-17$ & 67.55 & & $\mathrm{~V}$ \\
\hline & & & & & & & $09-28-17$ & 69.74 & & $\mathrm{~V}$ \\
\hline & & & & & & & $11-27-17$ & 70.63 & & $\mathrm{~V}$ \\
\hline & & & & & & & $01-25-18$ & 68.59 & & $\mathrm{~V}$ \\
\hline & & & & & & & $03-20-18$ & 68.86 & & $\mathrm{~V}$ \\
\hline & & & & & & & $05-30-18$ & 69.32 & & $\mathrm{~V}$ \\
\hline & & & & & & & $07-25-18$ & 71.58 & & $\mathrm{~V}$ \\
\hline & & & & & & & $09-28-17$ & 84.56 & & $\mathrm{~V}$ \\
\hline & & & & & & & $11-22-17$ & 84.65 & & V \\
\hline & & & & & & & 01-29-18 & 82.11 & & $\mathrm{~V}$ \\
\hline & & & & & & & 03-20-18 & 82.33 & & $\mathrm{~V}$ \\
\hline & & & & & & & $05-30-18$ & 83.03 & & $\mathrm{~V}$ \\
\hline & & & & & & & $07-25-18$ & 84.63 & & $\mathrm{~V}$ \\
\hline & & & & & & & $09-25-18$ & 85.85 & & $\mathrm{~V}$ \\
\hline 22N-04W-26M02 & 472159123094001 & 47.367 & -123.161 & $06-25-02$ & 112 & 63 & $09-21-16$ & 7.43 & $\mathrm{R}$ & $\mathrm{T}$ \\
\hline & & & & & & & $12-01-16$ & 5.13 & & $\mathrm{~T}$ \\
\hline & & & & & & & $01-27-17$ & 4.29 & & $\mathrm{~T}$ \\
\hline & & & & & & & 03-29-17 & 0.90 & & $\mathrm{~T}$ \\
\hline & & & & & & & $05-30-17$ & -0.30 & & $\mathrm{~T}$ \\
\hline & & & & & & & $07-31-17$ & 0.85 & & $\mathrm{~V}$ \\
\hline & & & & & & & $10-02-17$ & 14.23 & & $\mathrm{~V}$ \\
\hline & & & & & & & $11-28-17$ & 3.80 & & $\mathrm{~V}$ \\
\hline & & & & & & & $01-26-18$ & 3.43 & & $\mathrm{~V}$ \\
\hline & & & & & & & 03-23-18 & 3.14 & & $\mathrm{~V}$ \\
\hline & & & & & & & 06-01-18 & 5.00 & $\mathrm{R}$ & $\mathrm{V}$ \\
\hline
\end{tabular}


Table 2. Measured monthly groundwater levels, Mason County, western Washington, 2016-18.-Continued

\begin{tabular}{|c|c|c|c|c|c|c|c|c|c|c|}
\hline \multirow{2}{*}{ Local well No. } & \multirow{2}{*}{ Site No. } & \multirow{2}{*}{ Latitude } & \multirow{2}{*}{ Longitude } & \multirow{2}{*}{$\begin{array}{l}\text { Date of well } \\
\text { construction }\end{array}$} & \multirow{2}{*}{$\begin{array}{c}\text { Well } \\
\text { depth } \\
\text { (ft) }\end{array}$} & \multirow{2}{*}{$\begin{array}{l}\text { Land- } \\
\text { surface } \\
\text { altitude } \\
\text { (ft) }\end{array}$} & \multirow{2}{*}{$\begin{array}{c}\text { Measure- } \\
\text { ment } \\
\text { date }\end{array}$} & \multirow{2}{*}{$\begin{array}{c}\text { Groundwater } \\
\text { level } \\
\text { (feet below } \\
\text { land-surface) }\end{array}$} & \multicolumn{2}{|c|}{ Groundwater level } \\
\hline & & & & & & & & & Status & Method \\
\hline \multirow[t]{13}{*}{$22 \mathrm{~N}-01 \mathrm{~W}-20 \mathrm{P} 01$} & 472235122495901 & 47.376 & -122.834 & 04-01-61 & 70 & 22 & $09-22-16$ & 5.35 & & $\mathrm{~T}$ \\
\hline & & & & & & & $11-28-16$ & 5.38 & $\mathrm{R}$ & $\mathrm{T}$ \\
\hline & & & & & & & $01-27-17$ & 6.74 & $\mathrm{R}$ & $\mathrm{T}$ \\
\hline & & & & & & & $03-28-17$ & 5.49 & & $\mathrm{~T}$ \\
\hline & & & & & & & $05-25-17$ & 6.11 & & $\mathrm{~V}$ \\
\hline & & & & & & & $07-24-17$ & 7.11 & & $\mathrm{~V}$ \\
\hline & & & & & & & $09-28-17$ & 5.99 & $\mathrm{R}$ & $\mathrm{V}$ \\
\hline & & & & & & & $11-22-17$ & 3.87 & & V \\
\hline & & & & & & & $01-29-18$ & 4.78 & & $\mathrm{~V}$ \\
\hline & & & & & & & $03-22-18$ & 5.41 & & $\mathrm{~V}$ \\
\hline & & & & & & & $05-30-18$ & 6.82 & & $\mathrm{~V}$ \\
\hline & & & & & & & $07-25-18$ & 7.60 & & V \\
\hline & & & & & & & $09-25-18$ & 7.36 & & V \\
\hline \multirow{13}{*}{$22 \mathrm{~N}-01 \mathrm{~W}-20 \mathrm{P} 02$} & 472240122495501 & 47.376 & -122.834 & $08-22-77$ & 69 & 21 & $09-22-16$ & 5.81 & & $\mathrm{~T}$ \\
\hline & & & & & & & $11-28-16$ & 6.07 & $\mathrm{R}$ & $\mathrm{T}$ \\
\hline & & & & & & & $01-27-17$ & 5.87 & & $\mathrm{~T}$ \\
\hline & & & & & & & $03-28-17$ & 6.49 & & $\mathrm{~T}$ \\
\hline & & & & & & & $05-25-17$ & 7.11 & & $\mathrm{~V}$ \\
\hline & & & & & & & $07-24-17$ & 7.98 & & V \\
\hline & & & & & & & $09-28-17$ & 6.47 & $\mathrm{R}$ & V \\
\hline & & & & & & & $11-22-17$ & 4.11 & & $\mathrm{~V}$ \\
\hline & & & & & & & $01-29-18$ & 5.51 & & V \\
\hline & & & & & & & $03-22-18$ & 6.18 & $\mathrm{R}$ & $\mathrm{V}$ \\
\hline & & & & & & & $05-30-18$ & 7.73 & $\mathrm{R}$ & $\mathrm{V}$ \\
\hline & & & & & & & $07-25-18$ & 8.41 & & V \\
\hline & & & & & & & 09-25-18 & 8.17 & & $\mathrm{~V}$ \\
\hline \multirow[t]{13}{*}{$22 \mathrm{~N}-02 \mathrm{~W}-14 \mathrm{~F} 01$} & 472348122535401 & 47.397 & -122.900 & $05-29-75$ & 97 & 18 & $09-22-16$ & 12.52 & & $\mathrm{~T}$ \\
\hline & & & & & & & $11-28-16$ & 10.29 & & $\mathrm{~T}$ \\
\hline & & & & & & & $01-30-17$ & 12.14 & & $\mathrm{~T}$ \\
\hline & & & & & & & $03-28-17$ & 10.77 & & $\mathrm{~T}$ \\
\hline & & & & & & & $05-26-17$ & 12.80 & & $\mathrm{~T}$ \\
\hline & & & & & & & $07-31-17$ & 12.17 & & V \\
\hline & & & & & & & $09-28-17$ & 11.77 & & V \\
\hline & & & & & & & $11-27-17$ & 10.53 & & $\mathrm{~V}$ \\
\hline & & & & & & & $01-29-18$ & 10.08 & & V \\
\hline & & & & & & & 03-23-18 & 12.27 & & $\mathrm{~V}$ \\
\hline & & & & & & & $05-30-18$ & 13.24 & & $\mathrm{~V}$ \\
\hline & & & & & & & $07-24-18$ & 12.65 & & V \\
\hline & & & & & & & $09-25-18$ & 13.39 & & $\mathrm{~V}$ \\
\hline \multirow[t]{13}{*}{ 22N-05W-09G02 } & 472444123192001 & 47.412 & -123.322 & $03-16-88$ & 45.5 & 572 & $09-20-16$ & 14.43 & & $\mathrm{~T}$ \\
\hline & & & & & & & $11-30-16$ & 9.80 & & $\mathrm{~T}$ \\
\hline & & & & & & & $02-1-17$ & 10.99 & & $\mathrm{~T}$ \\
\hline & & & & & & & $03-27-17$ & 9.57 & & $\mathrm{~T}$ \\
\hline & & & & & & & $05-24-17$ & 11.67 & & $\mathrm{~V}$ \\
\hline & & & & & & & $07-19-17$ & 13.73 & & $\mathrm{~V}$ \\
\hline & & & & & & & $09-27-17$ & 14.43 & & $\mathrm{~V}$ \\
\hline & & & & & & & $11-30-17$ & 9.82 & & $\mathrm{~V}$ \\
\hline & & & & & & & 01-31-18 & 8.69 & & $\mathrm{~V}$ \\
\hline & & & & & & & 03-19-18 & 11.40 & & $\mathrm{~V}$ \\
\hline & & & & & & & $05-29-18$ & 13.04 & & $\mathrm{~V}$ \\
\hline & & & & & & & $07-23-18$ & 14.53 & & $\mathrm{~V}$ \\
\hline & & & & & & & $09-24-18$ & 13.77 & & V \\
\hline
\end{tabular}




\section{Groundwater and Surface-Water Data Collection for Mason County, Western Washington, 2016-18}

Table 2. Measured monthly groundwater levels, Mason County, western Washington, 2016-18.-Continued

\begin{tabular}{|c|c|c|c|c|c|c|c|c|c|c|}
\hline \multirow{2}{*}{ Local well No. } & \multirow{2}{*}{ Site No. } & \multirow{2}{*}{ Latitude } & \multirow{2}{*}{ Longitude } & \multirow{2}{*}{$\begin{array}{l}\text { Date of well } \\
\text { construction }\end{array}$} & \multirow{2}{*}{$\begin{array}{c}\text { Well } \\
\text { depth } \\
\text { (ft) }\end{array}$} & \multirow{2}{*}{$\begin{array}{l}\text { Land- } \\
\text { surface } \\
\text { altitude } \\
\text { (ft) }\end{array}$} & \multirow{2}{*}{$\begin{array}{c}\text { Measure- } \\
\text { ment } \\
\text { date }\end{array}$} & \multirow{2}{*}{$\begin{array}{c}\text { Groundwater } \\
\text { level } \\
\text { (feet below } \\
\text { land-surface) }\end{array}$} & \multicolumn{2}{|c|}{ Groundwater level } \\
\hline & & & & & & & & & Status & Method \\
\hline \multirow[t]{8}{*}{$22 \mathrm{~N}-01 \mathrm{~W}-07 \mathrm{~F} 01$} & 472450122512701 & 47.414 & -122.857 & $06-06-14$ & 42 & 308 & $09-22-16$ & 28.46 & & $\mathrm{~T}$ \\
\hline & & & & & & & $11-28-16$ & 23.74 & & $\mathrm{~T}$ \\
\hline & & & & & & & $01-30-17$ & 16.14 & & $\mathrm{~T}$ \\
\hline & & & & & & & $07-24-17$ & 23.65 & & $\mathrm{~V}$ \\
\hline & & & & & & & $09-28-17$ & 27.13 & & $\mathrm{~V}$ \\
\hline & & & & & & & $11-22-17$ & 27.47 & & $\mathrm{~V}$ \\
\hline & & & & & & & $01-25-18$ & 14.30 & & $\mathrm{~V}$ \\
\hline & & & & & & & $03-22-18$ & 18.93 & & $\mathrm{~V}$ \\
\hline \multirow{10}{*}{$22 \mathrm{~N}-01 \mathrm{~W}-08 \mathrm{D} 02$} & & & & & & & $11-28-16$ & 269.26 & & $\mathrm{~T}$ \\
\hline & & & & & & & $01-30-17$ & 280.90 & & $\mathrm{~T}$ \\
\hline & & & & & & & $03-28-17$ & 275.41 & & $\mathrm{~T}$ \\
\hline & & & & & & & $07-31-17$ & 298.08 & & $\mathrm{~V}$ \\
\hline & & & & & & & $09-28-17$ & 287.64 & & $\mathrm{~V}$ \\
\hline & & & & & & & $11-27-17$ & 270.13 & & $\mathrm{~V}$ \\
\hline & & & & & & & 01-29-18 & 277.82 & & $\mathrm{~V}$ \\
\hline & & & & & & & $03-23-18$ & 273.25 & & $\mathrm{~V}$ \\
\hline & & & & & & & $05-30-18$ & 285.81 & $\mathrm{R}$ & $\mathrm{V}$ \\
\hline & & & & & & & $09-25-18$ & 272.97 & & $\mathrm{~V}$ \\
\hline \multirow[t]{6}{*}{$22 \mathrm{~N}-05 \mathrm{~W}-09 \mathrm{C} 01$} & 472455123191001 & 47.415 & -123.320 & $10-31-03$ & 163 & 598 & $09-20-16$ & 22.20 & & $\mathrm{~T}$ \\
\hline & & & & & & & 01-31-18 & 18.87 & & $\mathrm{~V}$ \\
\hline & & & & & & & 03-19-18 & 20.81 & & $\mathrm{~V}$ \\
\hline & & & & & & & $05-29-18$ & 21.33 & & $\mathrm{~V}$ \\
\hline & & & & & & & $07-23-18$ & 22.04 & & $\mathrm{~V}$ \\
\hline & & & & & & & $09-24-18$ & 22.26 & & $\mathrm{~V}$ \\
\hline \multirow[t]{13}{*}{$22 \mathrm{~N}-04 \mathrm{~W}-09 \mathrm{C} 01$} & 472502123115801 & 47.417 & -123.199 & 09-05-89 & 160 & 697 & $09-21-16$ & -0.06 & & $\mathrm{~T}$ \\
\hline & & & & & & & $12-01-16$ & 20.53 & $\mathrm{R}$ & $\mathrm{T}$ \\
\hline & & & & & & & $01-30-17$ & 0.01 & & $\mathrm{~T}$ \\
\hline & & & & & & & $03-29-17$ & 2.49 & $\mathrm{R}$ & $\mathrm{T}$ \\
\hline & & & & & & & $05-30-17$ & 0.10 & $\mathrm{P}$ & $\mathrm{T}$ \\
\hline & & & & & & & 08-01-17 & 45.94 & $\mathrm{R}$ & $\mathrm{V}$ \\
\hline & & & & & & & 09-29-17 & 7.01 & & $\mathrm{~V}$ \\
\hline & & & & & & & $12-01-17$ & 0.04 & & $\mathrm{~V}$ \\
\hline & & & & & & & 01-31-18 & 0.05 & & $\mathrm{~V}$ \\
\hline & & & & & & & $03-30-18$ & 7.07 & $\mathrm{R}$ & $\mathrm{V}$ \\
\hline & & & & & & & 06-01-18 & 5.77 & & $\mathrm{~V}$ \\
\hline & & & & & & & 07-24-18 & 0.14 & & $\mathrm{~V}$ \\
\hline & & & & & & & 09-26-18 & 0.12 & & $\mathrm{~V}$ \\
\hline
\end{tabular}


Table 2. Measured monthly groundwater levels, Mason County, western Washington, 2016-18.-Continued

\begin{tabular}{|c|c|c|c|c|c|c|c|c|c|c|}
\hline \multirow{2}{*}{ Local well No. } & \multirow{2}{*}{ Site No. } & \multirow{2}{*}{ Latitude } & \multirow{2}{*}{ Longitude } & \multirow{2}{*}{$\begin{array}{l}\text { Date of well } \\
\text { construction }\end{array}$} & \multirow{2}{*}{$\begin{array}{l}\text { Well } \\
\text { depth } \\
\text { (ft) }\end{array}$} & \multirow{2}{*}{$\begin{array}{l}\text { Land- } \\
\text { surface } \\
\text { altitude } \\
\text { (ft) }\end{array}$} & \multirow{2}{*}{$\begin{array}{c}\text { Measure- } \\
\text { ment } \\
\text { date }\end{array}$} & \multirow{2}{*}{$\begin{array}{c}\text { Groundwater } \\
\text { level } \\
\text { (feet below } \\
\text { land-surface) }\end{array}$} & \multicolumn{2}{|c|}{ Groundwater level } \\
\hline & & & & & & & & & Status & Method \\
\hline \multirow[t]{13}{*}{$22 \mathrm{~N}-05 \mathrm{~W}-04 \mathrm{~F} 01$} & 472531123192701 & 47.425 & -123.324 & $03-25-97$ & 121 & 795 & $09-20-16$ & 104.75 & & $\mathrm{~T}$ \\
\hline & & & & & & & $11-30-16$ & 93.90 & & $\mathrm{~T}$ \\
\hline & & & & & & & $02-01-17$ & 99.08 & & $\mathrm{~T}$ \\
\hline & & & & & & & $03-27-17$ & 95.84 & & $\mathrm{~T}$ \\
\hline & & & & & & & $05-24-17$ & 102.60 & & $\mathrm{~V}$ \\
\hline & & & & & & & $07-19-17$ & 103.28 & & $\mathrm{~V}$ \\
\hline & & & & & & & $09-27-17$ & 104.26 & & $\mathrm{~V}$ \\
\hline & & & & & & & $11-30-17$ & 94.54 & & $\mathrm{~V}$ \\
\hline & & & & & & & $01-31-18$ & 95.10 & & $\mathrm{~V}$ \\
\hline & & & & & & & $03-19-18$ & 102.30 & & $\mathrm{~V}$ \\
\hline & & & & & & & $05-29-18$ & 103.35 & & $\mathrm{~V}$ \\
\hline & & & & & & & $07-23-18$ & 104.05 & & $\mathrm{~V}$ \\
\hline & & & & & & & $09-24-18$ & 104.66 & & $\mathrm{~V}$ \\
\hline \multirow[t]{13}{*}{ 22N-04W-05G02 } & 472537123130501 & 47.427 & -123.218 & $04-20-88$ & 115 & 757 & $09-21-16$ & 20.34 & & $\mathrm{~T}$ \\
\hline & & & & & & & $12-01-16$ & 21.30 & & $\mathrm{~T}$ \\
\hline & & & & & & & $01-30-17$ & 34.18 & & $\mathrm{~T}$ \\
\hline & & & & & & & $03-29-17$ & 18.06 & & $\mathrm{~T}$ \\
\hline & & & & & & & $05-30-17$ & 12.30 & & $\mathrm{~T}$ \\
\hline & & & & & & & 08-01-17 & 11.27 & & $\mathrm{~V}$ \\
\hline & & & & & & & $09-29-17$ & 20.06 & & $\mathrm{~V}$ \\
\hline & & & & & & & $12-01-17$ & 19.28 & & V \\
\hline & & & & & & & 01-31-18 & 29.61 & & $\mathrm{~V}$ \\
\hline & & & & & & & 03-30-18 & 26.11 & & V \\
\hline & & & & & & & 06-01-18 & 11.85 & & $\mathrm{~V}$ \\
\hline & & & & & & & $07-24-18$ & 14.74 & $\mathrm{R}$ & $\mathrm{V}$ \\
\hline & & & & & & & 09-26-18 & 18.65 & & $\mathrm{~V}$ \\
\hline \multirow{13}{*}{$23 \mathrm{~N}-03 \mathrm{~W}-31 \mathrm{E} 03$} & 472628123071101 & 47.441 & -123.120 & $11-30-90$ & 56 & 16 & $09-21-16$ & 10.18 & & $\mathrm{~T}$ \\
\hline & & & & & & & $12-01-16$ & 5.30 & & $\mathrm{~T}$ \\
\hline & & & & & & & $01-31-17$ & 6.30 & & $\mathrm{~T}$ \\
\hline & & & & & & & 03-29-17 & 5.12 & & $\mathrm{~T}$ \\
\hline & & & & & & & $05-30-17$ & 9.90 & & $\mathrm{~T}$ \\
\hline & & & & & & & $07-31-17$ & 11.69 & & $\mathrm{~V}$ \\
\hline & & & & & & & $09-29-17$ & 10.77 & & V \\
\hline & & & & & & & $11-28-17$ & 4.52 & & $\mathrm{~V}$ \\
\hline & & & & & & & $01-26-18$ & 4.41 & & $\mathrm{~V}$ \\
\hline & & & & & & & 03-30-18 & 8.19 & & $\mathrm{~V}$ \\
\hline & & & & & & & $06-01-18$ & 10.94 & & $\mathrm{~V}$ \\
\hline & & & & & & & $07-24-18$ & 12.29 & & V \\
\hline & & & & & & & $09-27-18$ & 11.59 & & $\mathrm{~V}$ \\
\hline \multirow{14}{*}{$23 \mathrm{~N}-03 \mathrm{~W}-19 \mathrm{~K} 01$} & 472804123063901 & 47.468 & -123.111 & $08-10-00$ & 103 & 173 & $09-21-16$ & 51.20 & & $\mathrm{~T}$ \\
\hline & & & & & & & $12-01-16$ & 50.22 & & $\mathrm{~T}$ \\
\hline & & & & & & & $01-26-17$ & 50.06 & & $\mathrm{~V}$ \\
\hline & & & & & & & $01-27-17$ & 50.44 & & $\mathrm{~T}$ \\
\hline & & & & & & & $03-29-17$ & 49.92 & & $\mathrm{~T}$ \\
\hline & & & & & & & $05-30-17$ & 50.50 & & $\mathrm{~T}$ \\
\hline & & & & & & & $07-31-17$ & 92.00 & $\mathrm{R}$ & V \\
\hline & & & & & & & $09-29-17$ & 50.99 & & $\mathrm{~V}$ \\
\hline & & & & & & & $11-28-17$ & 50.27 & & $\mathrm{~V}$ \\
\hline & & & & & & & 01-26-18 & 50.06 & & V \\
\hline & & & & & & & $03-23-18$ & 50.53 & & $\mathrm{~V}$ \\
\hline & & & & & & & 06-01-18 & 51.93 & & V \\
\hline & & & & & & & $07-24-18$ & 52.60 & & $\mathrm{~V}$ \\
\hline & & & & & & & $09-26-18$ & 53.46 & $\mathrm{R}$ & $\mathrm{V}$ \\
\hline
\end{tabular}


Table 2. Measured monthly groundwater levels, Mason County, western Washington, 2016-18._-Continued

\begin{tabular}{|c|c|c|c|c|c|c|c|c|c|c|}
\hline \multirow{2}{*}{ Local well No. } & \multirow{2}{*}{ Site No. } & \multirow{2}{*}{ Latitude } & \multirow{2}{*}{ Longitude } & \multirow{2}{*}{$\begin{array}{l}\text { Date of well } \\
\text { construction }\end{array}$} & \multirow{2}{*}{$\begin{array}{c}\text { Well } \\
\text { depth } \\
\text { (ft) }\end{array}$} & \multirow{2}{*}{$\begin{array}{l}\text { Land- } \\
\text { surface } \\
\text { altitude } \\
\text { (ft) }\end{array}$} & \multirow{2}{*}{$\begin{array}{c}\text { Measure- } \\
\text { ment } \\
\text { date }\end{array}$} & \multirow{2}{*}{$\begin{array}{c}\text { Groundwater } \\
\text { level } \\
\text { (feet below } \\
\text { land-surface) }\end{array}$} & \multicolumn{2}{|c|}{ Groundwater level } \\
\hline & & & & & & & & & Status & Method \\
\hline \multirow[t]{8}{*}{ 23N-04W-09Q01 } & 472937123114601 & 47.494 & -123.196 & 04-09-16 & 36.5 & 851 & $09-21-16$ & 17.70 & & $\mathrm{~T}$ \\
\hline & & & & & & & $12-01-16$ & 3.99 & $\mathrm{~T}$ & $\mathrm{~T}$ \\
\hline & & & & & & & $01-30-17$ & 5.93 & & $\mathrm{~T}$ \\
\hline & & & & & & & $08-01-17$ & 11.59 & & $\mathrm{~V}$ \\
\hline & & & & & & & $09-29-17$ & 16.08 & & $\mathrm{~V}$ \\
\hline & & & & & & & $11-28-17$ & 3.67 & & $\mathrm{~V}$ \\
\hline & & & & & & & $01-26-18$ & 3.21 & & $\mathrm{~V}$ \\
\hline & & & & & & & $03-23-18$ & 7.27 & & $\mathrm{~V}$ \\
\hline
\end{tabular}


Publishing support provided by the U.S. Geological Survey Science Publishing Network, Tacoma Publishing Service Center

For more information concerning the research in this report, contact the Director, Washington Water Science Center

U.S. Geological Survey

934 Broadway, Suite 300

Tacoma, Washington 98402

https://www.usgs.gov/centers/wa-water 
